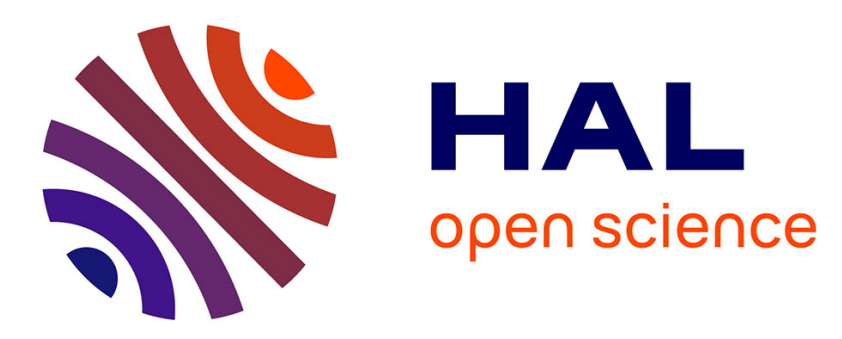

\title{
Formulation and numerical implementation of a constitutive law for concrete with strain-based damage and plasticity
}

Ulrich Häussler-Combe, Jens Hartig

\section{- To cite this version:}

Ulrich Häussler-Combe, Jens Hartig. Formulation and numerical implementation of a constitutive law for concrete with strain-based damage and plasticity. International Journal of Non-Linear Mechanics, 2008, 43 (5), pp.399. 10.1016/j.ijnonlinmec.2008.01.005 . hal-00501778

\section{HAL Id: hal-00501778 \\ https://hal.science/hal-00501778}

Submitted on 12 Jul 2010

HAL is a multi-disciplinary open access archive for the deposit and dissemination of scientific research documents, whether they are published or not. The documents may come from teaching and research institutions in France or abroad, or from public or private research centers.
L'archive ouverte pluridisciplinaire HAL, est destinée au dépôt et à la diffusion de documents scientifiques de niveau recherche, publiés ou non, émanant des établissements d'enseignement et de recherche français ou étrangers, des laboratoires publics ou privés. 


\section{Author's Accepted Manuscript}

INTERNATIONAL JOURNAL OF

NON-LINEAR MECHANICS

Formulation and numerical implementation of a constitutive law for concrete with strain-based damage and plasticity

Ulrich Häussler-Combe, Jens Hartig

PII: $\quad$ S0020-7462(08)00027-9

DOI: doi:10.1016/j.ijnonlinmec.2008.01.005

Reference: NLM 1445

To appear in: International Journal of NonLinear Mechanics

Received date: 7 February 2007

Revised date: 20 November 2007

Accepted date: 21 January 2008

Cite this article as: Ulrich Häussler-Combe and Jens Hartig, Formulation and numerical implementation of a constitutive law for concrete with strain-based damage and plasticity, International Journal of Non-Linear Mechanics (2008), doi:10.1016/j.ijnonlinmec.2008.01.005

This is a PDF file of an unedited manuscript that has been accepted for publication. As a service to our customers we are providing this early version of the manuscript. The manuscript will undergo copyediting, typesetting, and review of the resulting galley proof before it is published in its final citable form. Please note that during the production process errors may be discovered which could affect the content, and all legal disclaimers that apply to the journal pertain. 


\title{
Formulation and numerical implementation of a constitutive law for concrete with strain-based damage and plasticity
}

\author{
Ulrich Häussler-Combe, Jens Hartig \\ Institute of Concrete Structures, Technische Universität Dresden \\ 01062 Dresden, Germany
}

7th February 2008

\begin{abstract}
A triaxial constitutive law for concrete within the framework of isotropic damage combined with plasticity is proposed in this paper. It covers typical characteristics of concrete like nonlinear uniaxial compression and tension, bi- and triaxial failure criteria and dilatancy with a unified strain-based approach. Thus, this model is quite simple and especially suitable for strain-driven methods like common finite elements. It is complemented with a regularization method based on the crack band approach. A further issue is discussed with procedures for the model parameter determination for a wide range of concrete grades. The application of the model is demonstrated with typical benchmark tests for plain concrete.
\end{abstract}

\section{Keywords}

Concrete; Isotropic damage; Plasticity; Crack band model; Nonlinear analysis

*Phone +49-351-463-39586. Fax: +49-351-463-37279.

E-mail address: ulrich.haeussler-combe@tu-dresden.de. 


\section{Introduction}

A realistic three-dimensional description of concrete behavior is a basic requirement for a proper calculation of concrete structures or parts thereof under complex loading conditions, which arise in joints, edges, corners, generally bodies of arbitrary shape and under the action of concentrated loads. The same requirement also applies to severe actions like impact, penetration and blast on concrete structures. Such realistic models of material behavior have to agree with the results of experimental investigations, which have been performed in a large number for concrete during the last decades. Only a selection of them can be mentioned in the following. Uniaxial behavior is a major item and is well described in a number of technical codes. Beyond uniaxial strength basic investigations about biaxial strength were performed by [24]. Extended parameter domains and experimental techniques have been investigated by $[12,18,37]$ for the biaxial case. Triaxial experimental investigations have to be classified as confined with cylindrical specimen and two equal loading directions and truly triaxial with cubic specimen and three different loading directions. Confined triaxial states were investigated with triaxial cells $[19,22,28,33]$, whereas realization of truly triaxial states needs more elaborate facilities $[8,14,36,37]$. Major results of experimental investigations may be summarized as follows:

- While the biaxial compressive strength shows a moderate increase compared to the uniaxial compressive strength, the multiaxial compressive strength may become much higher depending on stress ratios.

- Approaching the material limits, i.e. the maximum sustainable stresses, the stress-strain behavior exhibits a pronounced nonlinearity.

- After achieving the material limits, a softening behavior arises, i.e. decreasing loading with increasing displacements. This is connected with localization phenomena, i.e. formation of crack bands or shear bands respectively.

- A pronounced dilatancy, i.e. a volume increase with increasing compression can be observed 
in the range near the material limits.

- While concrete in its initial state may be considered as isotropic, continuing loading leads to a load-induced anisotropy. This becomes especially evident with the development of cracks.

- Regarding unloading following loading, a reduced damaged stiffness can be observed compared to the initial stiffness. After a loading-unloading cycle with higher loads, plastic deformations remain in a stress-free state.

- A quasi-brittle behavior is observable with the development of cracks, as stress transfers may occur beyond emerging crack faces. As cracking is irreversible, this is connected with a considerable energy dissipation where a characteristic material property is given by the crack energy.

A wide range of model types has been used to describe concrete behavior, which may in a first approach be classified as microscopic, mesoscopic and macroscopic. While micro- and mesoscopic approaches distinguish the concrete constituents in different orders of resolution, macroscopic models assume a homogeneous material. This allows the application of the methods of classical continuum mechanics and makes macroscopic models suitable for calculations of whole structures.

Within continuum mechanics the constitutive theory provides plasticity and damage models for nonlinear behavior of materials. As the nonlinear behavior of concrete and other quasi-brittle materials is ruled only to a minor extent by slip of material lattices but more by nucleation and growth of micro-defects, damage models seem to be adequate under many aspects. Foundations of damage models have been laid by $[3,9,21,35]$ among others. Applications to concrete and other quasi-brittle materials were described, e.g., by $[29,30]$. Higher order damage tensors were introduced, see e.g. [26], to describe a load-induced material anisotropy, as it is especially characteristic for the tensile failure of concrete. An orthotropic approach was presented by [4], general anisotropic approaches among others by [13]. Proposals to include plastic deformations into damage models were given by, e.g., [16, 23,25]. While many particular aspects are covered by these alternatives, approaches combining altogether modeling quality, numerical costs, parameter determination pro- 
cedures and applicability in a well-balanced way seem to be rare.

In view of this target, isotropic damage in combination with plasticity is chosen as a foundation for a three-dimensional constitutive law for concrete. This formulation has some new aspects. It transforms a strength failure condition into a strain-based damage condition describing tension and compression behavior in a unified approach. This is supplemented with a new strain-based approach for plastic deformations. Both parts are combined in a new multiplicative form of a material tangent stiffness. As its formulation is based on strain, it may be easily implemented into commonly used strain-driven finite element procedures and it does not require iterations for fulfilling stress limit conditions on the integration point level. This also facilitates equilibrium iterations on the system level. Furthermore, a new kind of a crack band regularization procedure is described, with a simple approach based on equivalent crack energies and scaling of damage parameters in the softening range. The constitutive law is completed with procedures to determine its parameters for a wide range of concrete grades from generally available data. Finally, it is demonstrated that typical problems are accurately solved with these approaches. Thereby the following considerations will restrict to quasi-static short time behavior. Thus, long-time effects like creep and shrinkage will not be covered on the one hand, and on the other hand strength increase effects under dynamic short-time conditions at high strain rates are not included. Furthermore, cyclic loading cases with stiffness recovery and closing of microcracks will not be explicitly addressed.

The paper is organized as follows: To set the stage, the general form, the evolution of damage and plastic strains are given in Section 2 together with thermomechanical considerations, the formulation of a consistent tangent material stiffness and the discussion of computational aspects. This is extended with multiaxial limit states for stress and strain in Section 3. Uniaxial compressive and tensile behavior are derived as special cases in Section 4. These uniaxial cases in turn give the base to formulate procedures for the determination of material parameters in Section 5. A crack band approach for regularization is discussed in Section 6. Several numerical examples underline 
the applicability of the proposed constitutive law for concrete in Section 7, and finally Section 8 concludes the paper.

\section{An Isotropic Damage Law with Plasticity}

\subsection{General Form and Damage Evolution}

For isotropic damage with plasticity the following general constitutive form is assumed

$$
\begin{aligned}
& \boldsymbol{\sigma}=(1-D) \mathbf{E}: \boldsymbol{\epsilon}_{e d} \\
& \boldsymbol{\epsilon}_{e d}=\boldsymbol{\epsilon}-\boldsymbol{\epsilon}_{p}
\end{aligned}
$$

with the double contraction :, the Cauchy stress tensor $\boldsymbol{\sigma}$, a scalar damage parameter $D$, the elastic strain tensor $\boldsymbol{\epsilon}_{e d}$ subject to degradation, the total strain tensor $\boldsymbol{\epsilon}$, the plastic strain tensor $\boldsymbol{\epsilon}_{p}$ and the elastic tensor $\mathbf{E}$ with components

$$
E_{i j k l}=\Lambda \delta_{i j} \delta_{k l}+G\left(\delta_{i k} \delta_{j l}+\delta_{i l} \delta_{j k}\right), \quad \Lambda=\frac{\nu E}{(1+\nu)(1-2 \nu)}, \quad G=\frac{E}{2(1+\nu)}
$$

with the Lamé constants $\Lambda, G$ or the Young's modulus $E$ and Poisson's ratio $\nu$ respectively. The values $E, \nu$ are constant, while the damage parameter $D$ depends on the loading history and has a range $0 \leq D \leq 1$. A widely accepted approach for damage evolution of quasi-brittle materials like concrete is based on a Weibull distribution of microscopic defects [27]. This leads to a form

$$
D\left(\kappa_{d}\right)= \begin{cases}0 & \kappa_{d}<e_{d 0} \\ 1-\mathrm{e}^{-\left(\frac{\kappa_{d}-e_{d 0}}{e_{d}}\right)^{g_{d}}} & \kappa_{d} \geq e_{d 0}\end{cases}
$$

introducing a variable equivalent damage strain $\kappa_{d}$ and three material constants $e_{d}, e_{d 0}, g_{d}$. A typical damage evolution law is shown in Fig. 1 where $e_{d 0}$ marks a threshold value, $e_{d}$ rules how fast $D$ approaches 1 and $g_{d}$ is a shape parameter. It is assumed that the equivalent damage strain $\kappa_{d}$ amounts to the longitudinal elastic strain in uniaxial compression. Thus, in uniaxial compression Eq. (1) reduces to

$$
\sigma=-(1-D) E \kappa_{d}
$$

The damage approach has to be completed with a damage limit condition $F_{d}\left(\boldsymbol{\epsilon}_{e d}, \kappa_{d}\right)$, which gives $\kappa_{d}$ depending on arbitrary values of the elastic strain state $\boldsymbol{\epsilon}_{e d}$. It will be described in the following 
Section 3 leading to Eq. (57). Occurrence of damage is ruled by Kuhn-Tucker conditions

$$
F_{d} \leq 0, \quad \dot{\lambda}_{d} \geq 0, \quad \dot{\lambda}_{d} F_{d}=0
$$

with a damage multiplier $\lambda_{d}$. According to a framework given by [4] the damage multiplier equals the scalar damage parameter: $\lambda_{d}=D$. Hence, Eq. (5) leads to the consistency condition

$$
\mathbf{n}_{d}: \dot{\boldsymbol{\epsilon}}_{e d}-\dot{\lambda}_{d} H_{d} h_{d}=0, \quad \dot{\lambda}_{d}=\dot{D}=\frac{1}{H_{d} h_{d}} \mathbf{n}_{d}: \dot{\boldsymbol{\epsilon}}_{e d}
$$

with the gradient $\mathbf{n}_{d}=\partial F_{d} / \partial \boldsymbol{\epsilon}_{e d}$, see Eq. (58), and the hardening parameters $H_{d}=-\partial F_{d} / \partial \kappa_{d}$, $h_{d}=\partial \kappa_{d} / \partial \lambda_{d}$. The parameter $H_{d}$ can be derived from the damage limit condition $F_{d}$, see Eq. (57), and $h_{d}$ results from Eq. (3) with $\lambda_{d}=D$.

\subsection{Plastic Strains}

Regarding uniaxial compression, plastic behavior arises with a volume expansion or dilatancy respectively and results in remaining strains upon unloading. Here, a strain based formulation is chosen with a yield condition $F_{p}\left(\boldsymbol{\epsilon}_{e d}, \kappa_{e}\right)$ connecting the elastic strain tensor $\boldsymbol{\epsilon}_{e d}$ with an equivalent elastic strain $\kappa_{e}$. The yield condition separates elastic and plastic behavior and it is also used with Kuhn-Tucker conditions

$$
F_{p} \leq 0, \quad \dot{\lambda}_{p} \geq 0, \quad \dot{\lambda}_{p} F_{p}=0
$$

with a plastic multiplier $\lambda_{p}$. In a first approach, the yield condition should be simple. Thus, an isotropic form

$$
F_{p}\left(\boldsymbol{\epsilon}_{e d}, \kappa_{e}\right)=\frac{1}{2}\left(\boldsymbol{\epsilon}_{e d}^{+}: \boldsymbol{\epsilon}_{e d}^{+}+c_{c} \boldsymbol{\epsilon}_{e d}^{-}: \boldsymbol{\epsilon}_{e d}^{-}\right)-\frac{1}{2} \kappa_{e}^{2}
$$

similar to a von Mises condition is chosen here with the positive and negative projections of the elastic strains $\boldsymbol{\epsilon}_{e d}^{+}, \boldsymbol{\epsilon}_{e d}^{-}$. This approach distinguishes between tensile and compressive modes due to micro-cracking mechanisms [30]. The relation between these modes is determined by a further material parameter $c_{c}$, which accounts for the so called cross-effect. The positive projection $\mathbf{a}^{+}$of a second order symmetric tensor $\mathbf{a}$ is based on its spectral decomposition and is defined by

$$
a_{i j}^{+}=\sum_{(\alpha)} H\left(\lambda^{(\alpha)}\right) \lambda^{(\alpha)} v_{i}^{(\alpha)} v_{j}^{(\alpha)}
$$


with the Heaviside function $H$, the principal values $\lambda^{(\alpha)}$ of a and the principal directions with components $v_{i}^{(\alpha)}$. A corresponding relation holds for the negative projection with $-H\left(\lambda^{(\alpha)}\right)$ instead of $H\left(\lambda^{(\alpha)}\right)$. This results in $\mathbf{a}=\mathbf{a}^{+}+\mathbf{a}^{-}$.

As elastic strains should be accompanied with plastic strains under certain loading conditions, the equivalent elastic strain $\kappa_{e}$ has to be connected to a measure for plastic strains. Here, plastic strains are derived with associated plasticity by a flow rule

$$
\dot{\boldsymbol{\epsilon}}_{p}=\dot{\lambda}_{p} \mathbf{n}_{p}
$$

with the plastic multiplier $\dot{\lambda}_{p}$ and the gradient

$$
\mathbf{n}_{p}=\frac{\partial F_{p}}{\partial \boldsymbol{\epsilon}_{e d}}=\boldsymbol{\epsilon}_{e d}^{+}+c_{c} \boldsymbol{\epsilon}_{e d}^{-} .
$$

An equivalent plastic strain $\kappa_{p}$ is introduced as the length of the plastic strains

$$
\dot{\kappa}_{p}=\sqrt{\dot{\boldsymbol{\epsilon}}_{p}: \dot{\boldsymbol{\epsilon}}_{p}}=\dot{\lambda}_{p} \sqrt{\mathbf{n}_{p}: \mathbf{n}_{p}}
$$

It is assumed that the plastic equivalent strain is connected to the elastic equivalent strain by

$$
\kappa_{p}= \begin{cases}0 & \kappa_{e}<e_{p 0} \\ c_{p}\left(\kappa_{e}-e_{p 0}\right)\left(1-\mathrm{e}^{-\frac{\kappa_{e}-e_{p 0}}{e_{p}}}\right) & \kappa_{e} \geq e_{p 0}\end{cases}
$$

with three more material constants $c_{p}, e_{p}, e_{p 0}$ describing the material's plastic behavior. The qualitative relation between $\kappa_{p}$ and $\kappa_{e}$ is shown in Fig. 2. After $\kappa_{e}$ exceeds a threshold value $e_{p 0}$, the equivalent plastic strain $\kappa_{p}$ is proportional to $\kappa_{e}$ with a factor $c_{p}$ where $e_{p}$ rules the smooth transition. Comparing Eq. (13) with Eq. (3) the analogy can be seen, that $D$ and $\kappa_{p}$ result from the parameters $\kappa_{d}$ and $\kappa_{e}$ respectively. Furthermore, $\kappa_{d}$ and $\kappa_{e}$ both are related to the elastic strains by $F_{d}\left(\boldsymbol{\epsilon}_{e d}, \kappa_{d}\right)=0$ and $F_{p}\left(\boldsymbol{\epsilon}_{e d}, \kappa_{p}\right)=0$, respectively.

Now, the standard arguments are applied based on Eqs. (8), (12) and (13). Thus, Eq. (7) leads to a consistency condition

$$
\mathbf{n}_{p}: \dot{\boldsymbol{\epsilon}}_{e d}-\dot{\lambda}_{p} H_{p} h_{p}=\mathbf{n}_{p}: \dot{\boldsymbol{\epsilon}}-\dot{\lambda}_{p} \mathbf{n}_{p}: \mathbf{n}_{p}-\dot{\lambda}_{p} H_{p} h_{p}=0, \quad \dot{\lambda}_{p}=\frac{\mathbf{n}_{p}: \dot{\boldsymbol{\epsilon}}}{\mathbf{n}_{p}: \mathbf{n}_{p}+H_{p} h_{p}}
$$

with the hardening parameters $H_{p}=-\partial F_{p} / \partial \kappa_{e}=-\kappa_{e}$ and $h_{p}=\partial \kappa_{e} / \partial \lambda_{p}$. The parameter $h_{p}$ can be derived using Eq. (12)

$$
h_{p}=\frac{\mathrm{d} \kappa_{e}}{\mathrm{~d} \kappa_{p}} \frac{\mathrm{d} \kappa_{p}}{\mathrm{~d} \lambda_{p}}=\frac{\mathrm{d} \kappa_{e}}{\mathrm{~d} \kappa_{p}} \sqrt{\mathbf{n}_{p}: \mathbf{n}_{p}}
$$


where $\mathrm{d} \kappa_{e} / \mathrm{d} \kappa_{p}$ can be determined with Eq. (13) and $\mathbf{n}_{p}$ is given by Eq. (11). In summary, plastic strains are directly related to elastic and total strains by a system of first order ordinary differential equations, which is evident by combining Eqs. (10), (11) and (14). There is an indirect influence of the loading or the stress state respectively by relating elastic strains to stresses with Eq. (1).

\subsection{Thermodynamic Considerations}

Given a deformation history, a state is described here, beside $\boldsymbol{\epsilon}$, by the internal state variables $D$, and $\boldsymbol{\epsilon}_{p}$, which comprise the whole loading history. The variables $\boldsymbol{\epsilon}, \boldsymbol{\epsilon}_{p}, D$ completely describe the state of a material point, i.e. different points with the same state variable values have the same pair $\dot{\boldsymbol{\sigma}}, \dot{\boldsymbol{\epsilon}}$ for arbitrary selections of the strain rate $\dot{\boldsymbol{\epsilon}}$. The Helmholtz free energy $\Psi$ for the isothermal case is given by the energy, which is recovered with unloading from $\boldsymbol{\epsilon}$ to the state free of stresses.

Thus, $\Psi$ is given by

$$
\Psi=\frac{1-D}{2}\left(\boldsymbol{\epsilon}-\boldsymbol{\epsilon}_{p}\right): \mathbf{E}:\left(\boldsymbol{\epsilon}-\boldsymbol{\epsilon}_{p}\right)
$$

within this context, see, e.g., [6, Chapter 6.12]. This leads to $\boldsymbol{\sigma}=\partial \Psi / \partial \boldsymbol{\epsilon}$ in agreement with Eq. (1) and furthermore to generalized stresses $Y=\partial \Psi / \partial D=-\left(\boldsymbol{\epsilon}-\boldsymbol{\epsilon}_{p}\right): \mathbf{E}:\left(\boldsymbol{\epsilon}-\boldsymbol{\epsilon}_{p}\right) / 2$ and $\boldsymbol{\sigma}_{p}=\partial \Psi / \partial \boldsymbol{\epsilon}_{p}=-\boldsymbol{\sigma}$. The rate of the Helmholtz free energy is given by

$$
\dot{\Psi}=-\frac{\dot{D}}{2}\left(\boldsymbol{\epsilon}-\boldsymbol{\epsilon}_{p}\right): \mathbf{E}:\left(\boldsymbol{\epsilon}-\boldsymbol{\epsilon}_{p}\right)+\boldsymbol{\sigma}:\left(\dot{\boldsymbol{\epsilon}}-\dot{\boldsymbol{\epsilon}}_{p}\right) .
$$

The dissipation rate follows from

$$
\dot{d}=\boldsymbol{\sigma}: \dot{\boldsymbol{\epsilon}}-\dot{\Psi}=\frac{\dot{D}}{2}\left(\boldsymbol{\epsilon}-\boldsymbol{\epsilon}_{p}\right): \mathbf{E}:\left(\boldsymbol{\epsilon}-\boldsymbol{\epsilon}_{p}\right)+\boldsymbol{\sigma}: \dot{\boldsymbol{\epsilon}}_{p} .
$$

Regarding the Clausius-Duhem postulate, the inequality

$$
\dot{d} \geq 0
$$

has to be fulfilled. This inequality should hold for both parts of Eq. (18). This is obvious for the first damage part with $\dot{D} \geq 0$ and a positive definite structure of $\mathbf{E}$. Plastic strains have to be inspected for the second plastic part. Using Eqs. (1), (10) and (11) one arrives at

$$
\boldsymbol{\sigma}: \dot{\boldsymbol{\epsilon}}_{p}=\dot{\lambda}_{p}(1-D)\left(\boldsymbol{\epsilon}_{e d}: \mathbf{E}: \boldsymbol{\epsilon}_{e d}^{+}+c_{c} \boldsymbol{\epsilon}_{e d}: \mathbf{E}: \boldsymbol{\epsilon}_{e d}^{-}\right)
$$


With $\dot{\lambda}_{p} \geq 0,0 \leq D \leq 1, c_{c} \geq 0$ the tensor products remain to be inspected. Regarding Eq. (9) and the orthogonality of eigenvectors it may be shown that

$$
\boldsymbol{\epsilon}_{e d}: \mathbf{E}: \boldsymbol{\epsilon}_{e d}^{+}=\boldsymbol{\epsilon}_{e d}^{+}: \mathbf{E}: \boldsymbol{\epsilon}_{e d}^{+} \geq 0
$$

The same holds for the negative projection $\boldsymbol{\epsilon}_{e d}^{-}$. Finally, it follows $\boldsymbol{\sigma}: \dot{\boldsymbol{\epsilon}}_{p} \geq 0$ in accordance with the Clausius-Duhem inequality.

\subsection{Tangent Material Stiffness}

A consistent formulation of the tangent material stiffness is useful to reach convergence in Newton-

Raphson iteration schemes. Such a formulation is based on Eq. (1) differentiated with respect to time

$$
\dot{\boldsymbol{\sigma}}=-\dot{D} \mathbf{E}: \boldsymbol{\epsilon}_{e d}+(1-D) \mathbf{E}:\left(\dot{\boldsymbol{\epsilon}}-\dot{\boldsymbol{\epsilon}}_{p}\right)
$$

A vector

$$
\mathbf{m}_{d}=\frac{\epsilon_{e d}}{1-D}
$$

is introduced. Regarding a common framework, see [4], $\mathbf{m}_{d}$ describes damage strain directions in a general anisotropic stress based damage approach. It simplifies with isotropic damage and also has to be used for incremental stiffness relations in strain based damage. Thus, Eq. (22) leads to

$$
\dot{\boldsymbol{\sigma}}=(1-D) \mathbf{E}:\left(-\dot{D} \mathbf{m}_{d}+\dot{\boldsymbol{\epsilon}}-\dot{\boldsymbol{\epsilon}}_{p}\right)
$$

Using Eqs. (6), (10) and (14) this may be written as

$$
\begin{aligned}
& \dot{\boldsymbol{\sigma}}=\quad(1-D) \mathbf{E}:\left[\dot{\boldsymbol{\epsilon}}-\frac{\mathbf{m}_{d} \otimes \mathbf{n}_{d}}{H_{d} h_{d}}: \dot{\boldsymbol{\epsilon}}_{e d}-\frac{\mathbf{n}_{p} \otimes \mathbf{n}_{p}}{\mathbf{n}_{p}: \mathbf{n}_{p}+H_{p} h_{p}}: \dot{\boldsymbol{\epsilon}}\right] \\
& =(1-D) \mathbf{E}:\left[\dot{\boldsymbol{\epsilon}}-\frac{\mathbf{m}_{d} \otimes \mathbf{n}_{d}}{H_{d} h_{d}}:\left(\dot{\boldsymbol{\epsilon}}-\frac{\mathbf{n}_{p} \otimes \mathbf{n}_{p}}{\mathbf{n}_{p}: \mathbf{n}_{p}+H_{p} h_{p}}: \dot{\boldsymbol{\epsilon}}\right)-\frac{\mathbf{n}_{p} \otimes \mathbf{n}_{p}}{\mathbf{n}_{p}: \mathbf{n}_{p}+H_{p} h_{p}}: \dot{\boldsymbol{\epsilon}}\right] \\
& =\quad(1-D) \mathbf{E}:\left[\left(\mathcal{I}-\frac{\mathbf{m}_{d} \otimes \mathbf{n}_{d}}{H_{d} h_{d}}\right):\left(\mathcal{I}-\frac{\mathbf{n}_{p} \otimes \mathbf{n}_{p}}{\mathbf{n}_{p}: \mathbf{n}_{p}+H_{p} h_{p}}\right)\right]: \dot{\boldsymbol{\epsilon}}
\end{aligned}
$$

with the dyadic product $\otimes$ and the symmetric fourth order unit tensor $\mathcal{I}$ with components $\mathcal{I}_{\text {ijrs }}^{\text {sym }}=$ $\frac{1}{2}\left(\delta_{i r} \delta_{j s}+\delta_{i s} \delta_{j r}\right)$. The multiplicative form of the tangent material stiffness Eq. (25) is a new formulation, resulting from the dependence of damage limit $F_{d}$ and plastic limit $F_{p}$ on the elastic strains. It gives the tangent material stiffness in cases $\dot{\lambda}_{d}>0, \dot{\lambda}_{p}>0$ according to the KuhnTucker conditions Eqs. (5), (7). This has to be modified in cases without ongoing damage, without 
ongoing plastic strains, or without both. The tangent material stiffness is symmetric if $\mathbf{n}_{d}$ and $\mathbf{m}_{d}$ are coaxial, but this is not considered as mandatory [20, Chapter 23]. The properties of the tangent stiffness may be analyzed with respect to types of failure, as is demonstrated by [32] for multi-dissipative materials by a spectral analysis. This has to be devoted to further investigations.

\subsection{Computational Aspects}

So far, constitutive equations have been given as systems of first order ordinary differential equations depending on time. For a time $t^{n+1}$ the constitutive equations are summarized as follows

$$
\begin{aligned}
& \dot{\boldsymbol{\epsilon}}_{e d}^{n+1}=\dot{\boldsymbol{\epsilon}}^{n+1}-\dot{\lambda}_{p}^{n+1} \mathbf{n}_{p}\left(\boldsymbol{\epsilon}_{e d}^{n+1}, \kappa_{p}^{n+1}\right) \\
& \dot{\kappa}_{p}^{n+1}=\dot{\lambda}_{p}^{n+1} h_{p}\left(\boldsymbol{\epsilon}_{e d}^{n+1}, \kappa_{p}^{n+1}\right) \\
& 0=\mathbf{n}_{p}\left(\boldsymbol{\epsilon}_{e d}^{n+1}, \kappa_{p}^{n+1}\right): \dot{\boldsymbol{\epsilon}}_{e d}^{n+1}-H_{p}\left(\boldsymbol{\epsilon}_{e d}^{n+1}, \kappa_{p}^{n+1}\right) \dot{\kappa}_{p}^{n+1} \\
& \dot{\kappa}_{d}^{n+1}=\dot{D}^{n+1} h_{d}\left(\boldsymbol{\epsilon}_{e d}^{n+1}, \kappa_{d}^{n+1}\right) \\
& 0 \\
& =\mathbf{n}_{d}\left(\boldsymbol{\epsilon}_{e d}^{n+1}, \kappa_{d}^{n+1}\right): \dot{\boldsymbol{\epsilon}}_{e d}^{n+1}-H_{d}\left(\boldsymbol{\epsilon}_{e d}^{n+1}, \kappa_{d}^{n+1}\right) \dot{\kappa}_{d}^{n+1}
\end{aligned}
$$

where $\dot{\boldsymbol{\epsilon}}(t)$ is given as a driving part, $\mathbf{n}_{p}, h_{p}, H_{p}, \mathbf{n}_{d}, h_{d}, H_{d}$ are given as functions, and $\boldsymbol{\epsilon}_{e d}(t), \lambda_{p}(t), \kappa_{p}(t), D(t), \kappa_{d}(t)$ have to be determined. This may be numerically solved with an implicit backward Euler scheme with a time step $\Delta t$

$$
\begin{aligned}
& \dot{\boldsymbol{\epsilon}}_{e d}^{n+1}=\frac{1}{\Delta t}\left(\boldsymbol{\epsilon}_{e d}^{n+1}-\boldsymbol{\epsilon}_{e d}^{n}\right)=\frac{\Delta \boldsymbol{\epsilon}_{e d}}{\Delta t} \quad \rightarrow \quad \boldsymbol{\epsilon}_{e d}^{n+1}=\boldsymbol{\epsilon}_{e d}^{n}+\Delta \boldsymbol{\epsilon}_{e d} \\
& \dot{\lambda}_{p}^{n+1}=\frac{1}{\Delta t}\left(\lambda_{p}^{n+1}-\lambda_{p}^{n}\right) \quad=\frac{\Delta \lambda_{p}}{\Delta t} \quad \rightarrow \quad \lambda_{p}^{n+1}=\lambda_{p}^{n}+\Delta \lambda_{p} \\
& \dot{\kappa}_{p}^{n+1}=\frac{1}{\Delta t}\left(\kappa_{p}^{n+1}-\kappa_{p}^{n}\right) \quad=\frac{\Delta \kappa_{p}}{\Delta t} \quad \rightarrow \quad \kappa_{p}^{n+1}=\kappa_{p}^{n}+\Delta \kappa_{p} \\
& \dot{D}^{n+1}=\frac{1}{\Delta t}\left(D^{n+1}-D n\right)=\frac{\Delta D}{\Delta t} \quad \rightarrow \quad D^{n+1}=D^{n}+\Delta D \\
& \dot{\kappa}_{d}^{n+1}=\frac{1}{\Delta t}\left(\kappa_{d}^{n+1}-\kappa_{d}^{n}\right) \quad=\frac{\Delta \kappa_{d}}{\Delta t} \quad \rightarrow \quad \kappa_{d}^{n+1}=\kappa_{d}^{n}+\Delta \kappa_{d}
\end{aligned}
$$

leading to a system of nonlinear algebraic equations

$$
\begin{array}{rlr}
\mathbf{a}=\Delta \boldsymbol{\epsilon}_{e d}+\Delta \lambda_{p} \mathbf{n}_{p}^{n+1}-\Delta \boldsymbol{\epsilon} & =0 \\
b=\Delta \lambda_{p} h_{p}^{n+1}-\Delta \kappa_{p} & =0 \\
c=\mathbf{n}_{p}^{n+1}: \Delta \boldsymbol{\epsilon}_{e d}-H_{p}^{n+1} \Delta \kappa_{p} & =0 \\
d=\Delta D h_{d}^{n+1}-\Delta \kappa_{d} & =0 \\
e=\mathbf{n}_{d}^{n+1}: \Delta \boldsymbol{\epsilon}_{e d}-H_{d}^{n+1} \Delta \kappa_{d}=0
\end{array}
$$


with $\mathbf{n}_{p}^{n+1}=\mathbf{n}_{p}\left(\boldsymbol{\epsilon}_{e d}^{n}+\Delta \boldsymbol{\epsilon}_{e d}, \kappa_{p}^{n}+\Delta \kappa_{p}\right)$ and so on. For given values $\boldsymbol{\epsilon}_{e d}^{n}, \lambda_{p}^{n}, \kappa_{p}^{n}, D^{n}, \kappa_{d}^{n}$ the Eqs. (28) comprise scalar 10 equations for scalar 10 unknowns $\Delta \epsilon_{e d}, \Delta \lambda_{p}, \Delta \kappa_{p}, \Delta D, \Delta \kappa_{d}$ on the integration point level. It can be seen that the set of the last two equations describing damage evolution and the set of the first ones describing plasticity evolution are coupled by the elastic strains $\epsilon_{e d}$ only. This again results from $F_{d}, F_{p}$ depending on elastic strains and facilitates the simultaneous computation of damage and plasticity to a large extent.

Similar to the algorithms of stress-based plasticity [34, Chapter 3.6] the system

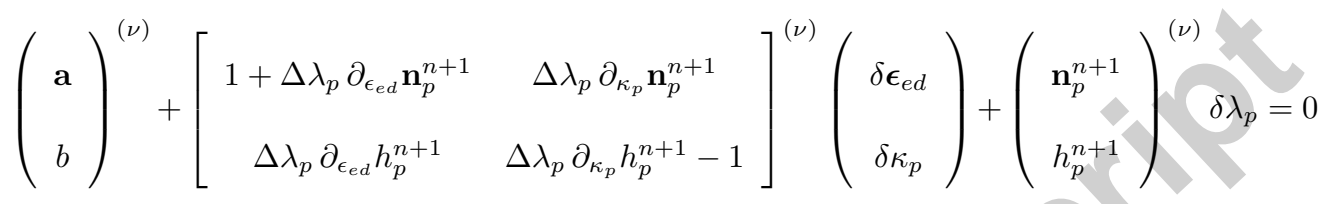

with

$$
c^{(\nu)}+\left[\begin{array}{ll}
\mathbf{n}_{p}^{n+1} & -H_{p}^{n+1}
\end{array}\right]^{(\nu)}\left(\begin{array}{c}
\delta \boldsymbol{\epsilon}_{e d} \\
\delta \kappa_{p}
\end{array}\right)=0
$$

may be used to iterate for increments of the elastic strains and plastic variables with a NewtonRaphson approach in the $(\nu)$-th iteration cycle. Time step increments are then given by

$$
\Delta \boldsymbol{\epsilon}_{e d}^{(\nu+1)}=\Delta \boldsymbol{\epsilon}_{e d}^{(\nu)}+\delta \boldsymbol{\epsilon}_{e d}, \quad \Delta \kappa_{p}^{(\nu+1)}=\Delta \kappa_{p}^{(\nu)}+\delta \kappa_{p}, \quad \Delta \lambda_{p}^{(\nu+1)}=\Delta \lambda_{p}^{(\nu)}+\delta \lambda_{p}
$$

As determination of the partial derivatives $\partial_{\epsilon_{e d}} \mathbf{n}_{p}, \partial_{\kappa_{p}} \mathbf{n}_{p}, \partial_{\epsilon_{e d}} h_{p}, \partial_{\kappa_{p}} h_{p}$ is elaborate and not mandatory, they may be disregarded in Eq. (29). This scheme also leads to convergent results for $\Delta \boldsymbol{\epsilon}_{e d}, \Delta \kappa_{p}, \Delta \lambda_{p}$ in all performed computations. With $\Delta \boldsymbol{\epsilon}_{e d}, \boldsymbol{\epsilon}_{e d}^{n+1}$ determined, the value of $\kappa_{d}^{n+1}$ can be directly computed from $F_{d}\left(\epsilon_{e d}^{n+1}, \kappa_{d}^{n+1}\right)=0$ without iteration, as will later become obvious with Eq. (57). Furthermore, $D^{n+1}$ can directly be computed with Eq. (3).

Finally, these values are used to update the stress with the implicit backward Euler scheme

$$
\dot{\boldsymbol{\sigma}}^{n+1}=\frac{1}{\Delta t}\left(\boldsymbol{\sigma}^{n+1}-\boldsymbol{\sigma}^{n}\right)=\frac{\Delta \boldsymbol{\sigma}}{\Delta t} \quad \rightarrow \quad \boldsymbol{\sigma}^{n+1}=\boldsymbol{\sigma}^{n}+\Delta \boldsymbol{\sigma}
$$

applied to Eq. (22) at time $t^{n+1}$

$$
\dot{\boldsymbol{\sigma}}^{n+1}=\left(1-D^{n+1}\right) \mathbf{E}: \dot{\boldsymbol{\epsilon}}_{e d}^{n+1}-\dot{D}^{n+1} \mathbf{E}: \boldsymbol{\epsilon}_{e d}^{n+1}
$$

leading to

$$
\Delta \boldsymbol{\sigma}=\left(1-D^{n+1}\right) \mathbf{E}: \Delta \boldsymbol{\epsilon}_{e d}-\Delta D \mathbf{E}: \boldsymbol{\epsilon}_{e d}^{n+1}
$$


The question of a tangent algorithmic stiffness, which generally is different to the tangent material stiffness Eq. (25), remains to be discussed. The tangent algorithmic modulus is defined as

$$
\mathbf{C}_{a l g}=\frac{\partial \Delta \sigma}{\partial \Delta \epsilon}
$$

To simplify the description, only the case with pure damage and $\boldsymbol{\epsilon}_{e d}=\boldsymbol{\epsilon}$ will be considered in this discussion. For the implicit Euler backward scheme this leads to

$$
\mathbf{C}_{a l g}^{n+1}=\mathbf{E}:\left[\left(1-D^{n+1}-\Delta D\right) \mathcal{I}-\frac{1}{h_{d}^{n+1} H_{d}^{n+1}}\left(\boldsymbol{\epsilon}^{n+1}+\Delta \boldsymbol{\epsilon}\right) \otimes \mathbf{n}_{d}^{n+1}\right]
$$

But the numerical calculations described in Section 7 show that this form does not improve the convergence compared to the corresponding tangent material stiffness applied at time $t^{n+1}$. This behavior remains to be investigated.

\section{$3 \quad$ Multiaxial Limit States}

\subsection{Principal Values}

As an isotropic material behavior is assumed, the principal stresses $\sigma_{1}, \sigma_{2}, \sigma_{3}$ and the principal strains $\epsilon_{1}, \epsilon_{2}, \epsilon_{3}$ with their coinciding directions are sufficient to characterize the material's state irrespective of their orientation in the material. Thus, in the following a notation

$$
\sigma_{1} \geq \sigma_{2} \geq \sigma_{3}, \quad \epsilon_{1} \geq \epsilon_{2} \geq \epsilon_{3}
$$

is used with positive tensile values and negative compressive values. In the case of tensile stresses, $\sigma_{1}$ denotes the largest principal tensile stress. An alternative form of principal stresses is given by the stress invariants

$$
\begin{array}{lcc}
I_{\boldsymbol{\sigma}, 1} & = & \sigma_{1}+\sigma_{2}+\sigma_{3} \\
J_{\boldsymbol{\sigma}, 2}= & \frac{1}{6}\left[\left(\sigma_{1}-\sigma_{2}\right)^{2}+\left(\sigma_{2}-\sigma_{3}\right)^{2}+\left(\sigma_{3}-\sigma_{1}\right)^{2}\right], \\
J_{\boldsymbol{\sigma}, 3}= & \left(\sigma_{1}-\sigma_{m}\right)\left(\sigma_{2}-\sigma_{m}\right)\left(\sigma_{3}-\sigma_{m}\right)
\end{array}
$$

with $\sigma_{m}=\left(\sigma_{1}+\sigma_{2}+\sigma_{3}\right) / 3$ where $J_{\boldsymbol{\sigma}, 2}, J_{\boldsymbol{\sigma}, 3}$ are the second and third invariant of the stress deviator. The principal stress space has characteristic elements: the hydrostatic axis $\sigma_{1}=\sigma_{2}=\sigma_{3}$, the deviatoric planes $\sigma_{1}+\sigma_{2}+\sigma_{3}=$ const. and the Rendulic plane spanned by the $\sigma_{1}$-axis and 
the line with $\sigma_{2}=\sigma_{3}$. A physical interpretation of a stress state $\sigma_{1}, \sigma_{2}, \sigma_{3}$ is given by the HaighWestergaard coordinates [5], which are defined by the length $\xi$ of the projection of $\sigma_{1}, \sigma_{2}, \sigma_{3}$ on the hydrostatic axis

$$
\xi \boldsymbol{\sigma}=\frac{1}{\sqrt{3}} I_{\boldsymbol{\sigma}, 1}=\sqrt{3} \sigma_{m}
$$

called hydrostatic length, the length $\rho$ of the projection of $\sigma_{1}, \sigma_{2}, \sigma_{3}$ on the deviatoric plane

$$
\rho_{\boldsymbol{\sigma}}=\sqrt{2 J_{\boldsymbol{\sigma}, 2}}
$$

called deviatoric length, and the so called deviatoric angle $\vartheta$

$$
\cos 3 \vartheta_{\boldsymbol{\sigma}}=\frac{3 \sqrt{3}}{2} \frac{J_{\boldsymbol{\sigma}, 3}}{J_{\boldsymbol{\sigma}, 2}^{3 / 2}}, \quad \vartheta_{\boldsymbol{\sigma}}=\frac{1}{3} \arccos \left(\frac{3 \sqrt{3}}{2} \frac{J_{\boldsymbol{\sigma}, 3}}{J_{\boldsymbol{\sigma}, 2}^{3 / 2}}\right)
$$

between the stress projection on the deviatoric plane and the intersection of deviatoric and Rendulic

plane. As stress states are constrained by Eq. (37), only a range $0^{\circ} \leq \vartheta \boldsymbol{\sigma} \leq 60^{\circ}$ has to be considered. Particular stress states are given by the compressive meridian $\sigma_{3}<\sigma_{2}=\sigma_{1}, \vartheta=60^{\circ}$ and the tensile meridian $\sigma_{3}=\sigma_{2}<\sigma_{1}, \vartheta=0^{\circ}$. Furthermore, uniaxial compression is described by

$$
\sigma_{1}=\sigma_{2}=0, \sigma_{3}<0 \rightarrow \xi \boldsymbol{\sigma}=\sigma_{3} / \sqrt{3}, \rho_{\boldsymbol{\sigma}}=\left|\sigma_{3}\right| \sqrt{2 / 3}, \vartheta_{\boldsymbol{\sigma}}=60^{\circ}
$$

uniaxial tension by

$$
\sigma_{2}=\sigma_{3}=0, \sigma_{1}>0 \rightarrow \xi \boldsymbol{\sigma}=\sigma_{1} / \sqrt{3}, \rho_{\boldsymbol{\sigma}}=\sigma_{1} \sqrt{2 / 3}, \vartheta_{\boldsymbol{\sigma}}=0^{\circ}
$$

and biaxial compression by

$$
\sigma_{1}=0, \sigma_{2}=\sigma_{3}<0 \rightarrow \xi_{\boldsymbol{\sigma}}=2 \sigma_{3} / \sqrt{3}, \rho_{\boldsymbol{\sigma}}=\left|\sigma_{3}\right| \sqrt{2 / 3}, \vartheta_{\boldsymbol{\sigma}}=0^{\circ}
$$

All these formulations may be transferred to the principal strain space with strain components $\epsilon_{i j}=\frac{1}{2}\left(\partial u_{i} / \partial x_{j}+\partial u_{j} / \partial x_{i}\right)$, in particular for $i \neq j$.

\subsection{A Limit State for Stresses}

A limit state is defined as state with maximum stresses. In the case of uniaxial compression, this leads to the uniaxial compressive strength $f_{c}$ (unsigned)

$$
f_{c}=\left(1-D_{l i m}\right) E \kappa_{d, l i m}, \quad D_{l i m}=D\left(\kappa_{d, l i m}\right)
$$


with Eq. (4) where $\kappa_{d, \text { lim }}$ amounts to the longitudinal elastic strain corresponding to the strength $f_{c}$. Generally, stress limits for isotropic materials may be described by surfaces in the principal stress space. The concrete limit surface is given by a curved tetrahedral form, opening in the negative octant. Such formulations have been proposed by $[10,17,31,38]$, a survey is given by [6]. In agreement with experimental data for concrete, all formulations share the same characteristics such as increasing deviatoric length of limit stresses with increasing hydrostatic length, slightly curved meridians and a "lower" tensile meridian compared to the compressive meridian. For the following, the proposal of [17] is chosen, which is formulated in the Haigh-Westergaards coordinates of limit stresses in a form

$$
F_{\sigma}=a_{1} \rho_{\boldsymbol{\sigma}}^{\prime 2}+\left(a_{2} \cos \vartheta_{\boldsymbol{\sigma}}+a_{3}\right) \rho_{\boldsymbol{\sigma}}^{\prime}+a_{4} \xi_{\boldsymbol{\sigma}}^{\prime}-1=0
$$

with $\rho_{\boldsymbol{\sigma}}^{\prime}=\rho_{\boldsymbol{\sigma}} / f_{c}, \xi_{\boldsymbol{\sigma}}^{\prime}=\xi \boldsymbol{\sigma} / f_{c}$ related to the uniaxial compressive strength $f_{c}$. Eq. (46) determines the deviatoric length, depending on the hydrostatic length and the deviatoric angle with a range $0^{\circ} \leq \vartheta_{\boldsymbol{\sigma}} \leq 60^{\circ}$. The remaining stress space is covered by symmetry operations based on the rule $\sigma_{1} \geq \sigma_{2} \geq \sigma_{3}$. Four parameters $a_{1}, \ldots a_{4}$ are introduced, which rule the exact form of the limit surface and may be used to reflect experimental data.

This approach has a drawback insofar as it is continuous but not smooth along the compressive meridians, see Fig. 3. Thus, as will be later seen with Eq. (58), the gradient $\mathbf{n}_{d}$ is not uniquely defined along these edges, which is joined with a non-unique tangent stiffness Eq. (25) on the compressive meridian. Problems of this type are addressed by [34, Chapter 5], where multiple plastic mechanisms are combined into a common tangent stiffness. An analogous procedure might be used here, by assuming the failure surface to be composed of three smooth parts intersecting in the compressive meridians. Such an approach remains to be investigated. In the present investigation the mean direction between the two gradients is used, if necessary. Regarding convergence of the equilibrium iteration, no difficulties occur with this form in the numerical calculations described in Section 7. 
In order to determine the material parameters $a_{1}, \ldots a_{4}$, particular limit states are regarded.

First of all uniaxial compression with $\sigma_{3}=-f_{c}$. Using Eq. (42) we have

$$
\xi_{\boldsymbol{\sigma}}^{\prime}=-\frac{1}{\sqrt{3}}, \quad \rho_{\boldsymbol{\sigma}}^{\prime}=\sqrt{\frac{2}{3}}, \quad \vartheta_{\boldsymbol{\sigma}}=60^{\circ}, \quad \cos \vartheta_{\boldsymbol{\sigma}}=\frac{1}{2} .
$$

Furthermore, uniaxial tensile strength is given with $\sigma_{1}=f_{c t}=\alpha_{1} f_{c}$. Using Eq. (43) leads to

$$
\xi_{\boldsymbol{\sigma}}^{\prime}=\frac{\alpha_{1}}{\sqrt{3}}, \quad \rho_{\boldsymbol{\sigma}}^{\prime}=\alpha_{1} \sqrt{\frac{2}{3}}, \quad \vartheta_{\boldsymbol{\sigma}}=0^{\circ}, \quad \cos \vartheta_{\boldsymbol{\sigma}}=1 .
$$

Then, biaxial compressive strength can be described with $\sigma_{2}=\sigma_{3}=-\alpha_{2} f_{c}$. With Eq. (44) we get

$$
\xi_{\boldsymbol{\sigma}}^{\prime}=-\frac{2 \alpha_{2}}{\sqrt{3}}, \quad \rho_{\boldsymbol{\sigma}}^{\prime}=\alpha_{2} \sqrt{\frac{2}{3}}, \quad \vartheta_{\boldsymbol{\sigma}}=0^{\circ}
$$

Finally, a limit state appearing in triaxial compression loading as usually applied in a triaxial cell for geomaterials and concrete is considered [33]. This is represented by $\sigma_{1}=\sigma_{2}=-\beta f_{c}, \sigma_{3}=-\alpha_{3} f_{c}$ with $\alpha_{3}>\beta$. In a similar manner as before this leads to

$$
\xi_{\boldsymbol{\sigma}}^{\prime}=-\left(2 \beta+\alpha_{3}\right) / \sqrt{3}, \quad \rho_{\boldsymbol{\sigma}}^{\prime}=\sqrt{2 / 3} \quad\left(\alpha_{3}-\beta\right), \quad \vartheta_{\boldsymbol{\sigma}}=60^{\circ}, \quad \cos \vartheta_{\boldsymbol{\sigma}}=\frac{1}{2}
$$

Several characteristics of the limit stress surface are thus described by the four parameters $\alpha_{1}, \alpha_{2}, \alpha_{3}, \beta$.

Their values can be derived from experiments. This will be discussed in Section 5. Applying the general limit stress condition Eq. (46) to each particular case leads to a system of equations

$$
\begin{aligned}
& 2 a_{1} / 3+\left(a_{2} / 2+a_{3}\right) \sqrt{2 / 3}-a_{4} / \sqrt{3} \quad=1 \\
& 2 a_{1} \alpha_{1}^{2} / 3+\left(a_{2}+a_{3}\right) \alpha_{1} \sqrt{2 / 3}+a_{4} \alpha_{1} / \sqrt{3} \quad=1
\end{aligned}
$$

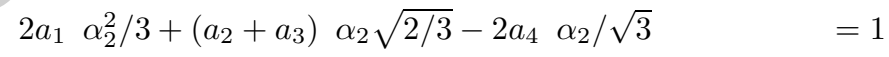

$$
\begin{aligned}
& 2 a_{1}\left(\alpha_{3}-\beta\right)^{2} / 3+\left(a_{2} / 2+a_{3}\right)\left(\alpha_{3}-\beta\right) \sqrt{2 / 3}-a_{4}\left(2 \beta+\alpha_{3}\right) / \sqrt{3}=1
\end{aligned}
$$

which can be solved for the parameters $a_{1}, \ldots a_{4}$ with given values $\alpha_{1}, \alpha_{2}, \alpha_{3}, \beta$.

\subsection{A Limit State for Strains}

As a prerequisite the constitutive law is reformulated. We use Eq. (1) for the first invariant of stresses and get from Eq. (38)

$$
I_{\boldsymbol{\sigma}, 1}=(1-D) \frac{E}{1-2 \nu} I_{\boldsymbol{\epsilon}_{e d, 1}}, \quad J_{\boldsymbol{\sigma}, 2}=(1-D)^{2} 4 G^{2} J_{\boldsymbol{\epsilon}_{e d, 2}}, \quad J_{\boldsymbol{\sigma}, 3}=(1-D)^{3} 8 G^{3} J_{\boldsymbol{\epsilon}_{e d, 3}}
$$


with the shear modulus $G=E /(2(1+\nu))$, the first invariant $I_{\boldsymbol{\epsilon}_{e d, 1}}$ of the elastic strain and the second and third invariant $J_{\boldsymbol{\epsilon}_{e d, 2}}, J_{\boldsymbol{\epsilon}_{e d, 3}}$ of the elastic strain deviator. This is used for the HaighWestergaard coordinates of stress with Eqs. (39)-(41), leading to

$$
\xi_{\boldsymbol{\sigma}}=\frac{(1-D) E}{1-2 \nu} \xi_{\boldsymbol{\epsilon}_{e d}}, \quad \rho \boldsymbol{\sigma}=(1-D) 2 G \rho_{\boldsymbol{\epsilon}_{e d}}, \quad \cos 3 \vartheta_{\boldsymbol{\sigma}}=\frac{3 \sqrt{3}}{2} \frac{J_{\boldsymbol{\sigma}, 3}}{J_{\boldsymbol{\sigma}, 2}^{3 / 2}}=\frac{3 \sqrt{3}}{2} \frac{J_{\boldsymbol{\epsilon}, 3}}{J_{\boldsymbol{\epsilon}, 2}^{3 / 2}}=\cos 3 \vartheta_{\boldsymbol{\epsilon}_{e d}}
$$

with the Haigh-Westergaard coordinates $\xi_{\boldsymbol{\epsilon}_{e d}}, \rho \boldsymbol{\epsilon}_{e d}, \vartheta \boldsymbol{\epsilon}_{e d}$ of the elastic strain. Thus, the stress limit condition Eq. (46) can be expressed as

$a_{1}\left[\left(1-D_{c r}\right) 2 G \rho_{\boldsymbol{\epsilon}_{e d}}\right]^{2}+f_{c}\left(a_{2} \cos \vartheta_{\boldsymbol{\epsilon}_{e d}}+a_{3}\right)\left(1-D_{l i m}\right) 2 G \rho_{\boldsymbol{\epsilon}_{e d}}+f_{c} a_{4} \frac{\left(1-D_{l i m}\right) E}{1-2 \nu} \xi_{\boldsymbol{\epsilon}_{e d}}-f_{c}^{2}=0$

with the damage value $D_{\text {lim }}$ for stress limit states. Considering a relation $\rho_{\mathbf{a}} \cos \vartheta_{\mathbf{a}}=\sqrt{3 / 2} a_{1}-$ $I_{\mathbf{a}, 1} / \sqrt{6}$ for every symmetric second order tensor a with a maximum principal value $a_{1}$, replacing the Haigh-Westergaard coordinates with the elastic strain invariants according to Eq. (38) and $2 G$ with $E /(1+\nu)$ and using Eq. (45) gives

$$
\begin{aligned}
F_{\epsilon} & =b_{1} \quad J_{\boldsymbol{\epsilon}_{e d, 2}}+\frac{f_{c}}{\left(1-D_{l i m}\right) E}\left[b_{2} \sqrt{J_{\boldsymbol{\epsilon}_{e d, 2}}}+b_{3} \epsilon_{e d, 1}+b_{4} I_{\boldsymbol{\epsilon}_{e d, 1}}\right]-\left[\frac{f_{c}}{\left(1-D_{l i m}\right) E}\right]^{2} \\
& =b_{1} J_{\boldsymbol{\epsilon}_{e d, 2}}+\kappa_{d, l i m}\left[b_{2} \sqrt{J_{\boldsymbol{\epsilon}_{e d, 2}}}+b_{3} \epsilon_{e d, 1}+b_{4} I_{\boldsymbol{\epsilon}_{e d, 1}}\right]-\kappa_{d, l i m}^{2}=0
\end{aligned}
$$

with the largest principal strain $\epsilon_{e d, 1}$ of $\epsilon_{e d}$ and

$$
\begin{aligned}
b_{1} & =\frac{2 a_{1}}{(1+\nu)^{2}}, \\
b_{2} & =\sqrt{2} \frac{a_{3}}{(1+\nu)}, \\
b_{3} & =\sqrt{\frac{3}{2}} \frac{a_{2}}{(1+\nu)}, \\
b_{4} & =\frac{1}{\sqrt{3}} \frac{a_{4}}{1-2 \nu}-\frac{1}{\sqrt{6}} \frac{a_{2}}{(1+\nu)} .
\end{aligned}
$$

Eq. (55) is a reformulation of the stress limit condition Eq. (46) in the strain space where $b_{1}, \ldots b_{4}$ are constant material parameters describing the shape of the strain surface with the damage value $D_{\text {lim }}$ or the equivalent critical damage strain $\kappa_{d, l i m}$ respectively. This is generalized for usage as damage limit condition with

$$
F_{d}\left(\boldsymbol{\epsilon}_{e d}, \kappa_{d}\right)=b_{1} J_{\boldsymbol{\epsilon}_{e d, 2}}+\kappa_{d}\left[b_{2} \sqrt{J_{\boldsymbol{\epsilon}_{e d, 2}}}+b_{3} \epsilon_{e d, 1}+b_{4} I_{\boldsymbol{\epsilon}_{e d, 1}}\right]-\kappa_{d}^{2}=0
$$

where $\kappa_{d, \text { lim }}$ has been replaced with $\kappa_{d}$. This particular form admits "increasing" stresses for $\kappa_{d}<\kappa_{d, \text { lim }}$ and "decreasing" stresses for $\kappa_{d}>\kappa_{d, \text { lim }}$, as $\kappa_{d}$ rules damage $D$ with Eq. (3) and 
damage rules stress with Eq. (1). Furthermore, this form should be isotropic with respect to rotations of the reference system. Finally, from Eq. (57) the gradient used for the tangent material stiffness Eq. (25) can be derived with

$$
\mathbf{n}_{d}=\frac{\partial F_{d}}{\partial \boldsymbol{\epsilon}_{e d}}=\left(b_{1}+b_{2} \frac{\kappa_{d}}{2 \sqrt{J_{\boldsymbol{\epsilon}_{e d, 2}}}}\right) \boldsymbol{\epsilon}_{e d}^{d e v}+\kappa_{d}\left(b_{3} \mathbf{d}_{1} \otimes \mathbf{d}_{1}+b_{4} \mathbf{I}\right)
$$

with the deviatoric part $\boldsymbol{\epsilon}_{e d}^{d e v}$ of the elastic strain $\boldsymbol{\epsilon}_{e d}$, the eigenvector $\mathbf{d}_{1}$ of $\epsilon_{e d, 1}$ and the second order unit tensor I with components $I_{i j}=\delta_{i j}$. The particular form chosen with Eq. (57) leads to an asymmetric stiffness, as $\mathbf{n}_{d}$ is not coaxial to $\mathbf{m}_{d}$, see Eq. (23).

\section{The Uniaxial Special Cases}

In uniaxial cases, principal directions coincide with the coordinate directions. Regarding the convention Eq. (37), uniaxial compression is characterized by $\sigma_{1}=\sigma_{2}=0, \sigma_{3}<0$ and $\epsilon_{e d, 3}<0$ leading to $\epsilon_{e d, 1}=\epsilon_{e d, 2}=-\nu \epsilon_{e d, 3}$ with Eq. (1) and the latter leading to $\kappa_{d}=-\epsilon_{e d, 3}$ with Eq. (57). The last result agrees with the assumptions concerning damage evolution Eq. (3). Thus, Eqs. (1), (3) give

$$
\sigma_{3}=\left\{\begin{array}{cc}
E\left(\epsilon_{3}-\epsilon_{p 3}\right) & \epsilon_{3}-\epsilon_{p 3}>e_{d 0} \\
\mathrm{e}^{-\left(\frac{-\left(\epsilon_{3}-\epsilon_{p 3}\right)-e_{d 0}}{e_{d}}\right)^{g_{d}}} E\left(\epsilon_{3}-\epsilon_{p 3}\right) & \epsilon_{3}-\epsilon_{p 3} \leq e_{d 0}
\end{array}\right.
$$

with the total and plastic longitudinal strains $\epsilon_{3}, \epsilon_{p 3}$. In this equation, $e_{d 0}, e_{d}, g_{d}$ are material parameters and $\epsilon_{3}$ may be prescribed as independent variable. The plastic strain $\epsilon_{p 3}$ remains to be determined in order to determine the stress $\sigma_{3}$. The case of ongoing plastic deformations is ruled by the yield Eq. (8). Taking uniaxial compression into account with

$$
\boldsymbol{\epsilon}_{e}^{+}=\left(\begin{array}{ccc}
-\nu_{0} \epsilon_{e d, 3} & -\nu_{0} \epsilon_{e d, 3} & 0
\end{array}\right)^{T}, \quad \boldsymbol{\epsilon}_{e}^{-}=\left(\begin{array}{lll}
0 & 0 & \epsilon_{e d, 3}
\end{array}\right)^{T}
$$

we get

$$
F_{p}=\frac{1}{2}\left(2 \nu^{2}+c_{c}\right) \epsilon_{e d, 3}^{2}-\frac{1}{2} \kappa_{e}^{2}=0
$$

which is resolved for the elastic equivalent strain

$$
\kappa_{e}=-c_{1} \epsilon_{e d, 3}, \quad c_{1}=\sqrt{2 \nu^{2}+c_{c}} .
$$


Combining this with Eq. (13) leads to the plastic equivalent strain depending on the longitudinal strains

$$
\kappa_{p}=c_{p}\left(-c_{1}\left(\epsilon_{3}-\epsilon_{p 3}\right)-e_{p 0}\right)\left(1-\mathrm{e}^{-\frac{-c_{1}\left(\epsilon_{3}-\epsilon_{p 3}\right)-e_{p 0}}{e_{p}}}\right), \quad \epsilon_{3}-\epsilon_{p 3}<\frac{e_{p 0}}{c_{1}}
$$

Furthermore, the plastic flow rule Eq. (10) together with Eq. (11) has to be taken into account. In the uniaxial compression case with a constant ratio of elastic strains, see Eq. (60), the plastic flow rule can be integrated to give the plastic strain components

$$
\epsilon_{p 1}=\epsilon_{p 2}=\bar{\lambda}_{p} \nu, \quad \epsilon_{p 3}=-\bar{\lambda}_{p} c_{c}
$$

with a scaled plastic multiplier $\bar{\lambda}_{p}$. The link between plastic strain components and equivalent plastic strain is given by Eq. (12), which now takes the form

$$
\kappa_{p}=c_{2} \bar{\lambda}_{p}, \quad c_{2}=\sqrt{2 \nu^{2}+c_{c}^{2}}
$$

Eqs. (63)-(65) lead to an equation for the longitudinal plastic strain

$$
\epsilon_{p 3}=-\frac{c_{c} c_{p}}{c_{2}}\left(-c_{1}\left(\epsilon_{3}-\epsilon_{p 3}\right)-e_{p 0}\right)\left(1-\mathrm{e}^{-\frac{-c_{1}\left(\epsilon_{3}-\epsilon_{p 3}\right)-e_{p 0}}{e_{p}}}\right), \quad \epsilon_{3}-\epsilon_{p 3}<\frac{e_{p 0}}{c_{1}}
$$

where $c_{1}, c_{2}, c_{c}, c_{p}, e_{p 0}, e_{p}$ are material constants and the total longitudinal strain $\epsilon_{3}$ is assumed to be given. Eqs. (59), (66) may be used to calculate the stress-strain relation for the uniaxial compression case. From Eq. (64) corresponding volumetric strains can be evaluated with

$$
\epsilon_{v o l}=\epsilon_{e d, 1}+\epsilon_{e d, 2}+\epsilon_{e d, 3}+\epsilon_{p 1}+\epsilon_{p 2}+\epsilon_{p 3}=(1-2 \nu)\left(\epsilon_{3}-\epsilon_{p 3}\right)+\left(1-\frac{2 \nu}{c_{c}}\right) \epsilon_{p 3}
$$

The other way round, Eqs. (59), (66), (67) can be used for the calibration of material parameters, which will be discussed in the following Section 5 .

Uniaxial behavior has to be completed with the tension case. Here we have $\sigma_{1}>0, \sigma_{2}=\sigma_{3}=0$ and $\epsilon_{e d, 3}>0$. Based on experience, plastic deformations can be neglected in the uniaxial tension case, which corresponds to the case $\kappa_{e}<e_{p 0}$ in Eq. (13). Thus, $\boldsymbol{\epsilon}_{e d}=\boldsymbol{\epsilon}$ will be assumed for uniaxial tension. Then Eqs. (1), (3) give

$$
\sigma_{1}=(1-D) E \epsilon_{1}=\mathrm{e}^{-\left(\frac{\kappa_{d}-e_{d 0}}{e_{d}}\right)^{g_{d}}} E \epsilon_{1}, \quad \epsilon_{2}=\epsilon_{3}=-\nu_{0} \epsilon_{1}
$$

This leads to decreasing lateral strains with continuously growing longitudinal strains, which is obviously not correct in case of tension cracking with cracks perpendicular to the tensile directions. 
This cannot be avoided with isotropic damage and has to be regarded as inherent drawback of this type of damage.

Considering the uniaxial tension strains for the damage limit condition Eq. (57) leads to

$$
F_{d}=b_{1} \frac{(1+\nu)^{2} \epsilon_{1}^{2}}{3}+\kappa_{d}\left(b_{2} \frac{1+\nu}{\sqrt{3}}+b_{3}+b_{4}(1-2 \nu)\right) \epsilon_{1}-\kappa_{d}^{2}
$$

With $F_{d}=0$ this may be solved for the positive value to give the equivalent damage strain for uniaxial tension depending on the longitudinal tensile strain

$$
\kappa_{d}=c_{3} \epsilon_{1}
$$

with a constant value $c_{3}$, which can be derived from the constant material parameters $\nu, b_{1} \ldots b_{4}$. This can be used with Eq. (68) to compute the stress-strain relation in the uniaxial tension case.

\section{Determination of Material Parameters}

As a basic requirement, a constitutive law for concrete should describe the nonlinear behavior under uniaxial compression. Regardless of any nonlinearities, the initial behavior is characterized by given values for the initial Young's modulus $E$ and Poisson's ratio $\nu$. Further characteristic values are given by the largest compressive stress, i.e. the compressive strength $f_{c}$ as an absolute value, and the corresponding strain $\epsilon_{c}$ as a negative value. A key role for uniaxial deformation behavior is given by the "cross-effect parameter" $c_{c}$. A value $c_{c}=0$ suppresses any longitudinal plastic strains under uniaxial compression, see Eqs. (10), (11). Increasing values $c_{c}>0$ lead to larger longitudinal plastic strains. A reasonable choice has a magnitude of $c_{c} \approx 0.1$. For further considerations, it proves to be appropriate to assume a value and to determine all remaining material parameters as will be discussed in the following. Further values of $c_{c}$ should then be tried in next iteration cycles if necessary. This approach first of all determines the values $c_{1}$, see Eq. (62), and $c_{2}$, see Eq. (65).

Another characteristic value of plastic behavior is given by a threshold value for the total longitudinal strain, when plasticity starts. This plastic threshold strain $\epsilon_{3}=\epsilon_{p, t h r}$ may be observed from experiments as fraction of the longitudinal strain $\epsilon_{c}$ with the maximum stress. With $\epsilon_{p 3}=0$ 
in Eq. (66) a relation

$$
e_{p 0}=-c_{1} \epsilon_{p, t h r}
$$

holds. In the following, it proves to be appropriate to choose a constant damage exponent $g_{d}=2$, see Eq. (3), for all concrete grades. The remaining set of damage material parameters $e_{d}, e_{d 0}$, see Eq. (3), and the set of plastic material parameters $c_{p}, e_{p}$, see Eq. (13), reveal some dependencies, which have to be resolved in a subiteration. This subiteration is marked by the upper index $i$ with

$$
\epsilon_{e d, c}^{i}+\epsilon_{p, c}^{i}=\epsilon_{c}
$$

in which the longitudinal strain $\epsilon_{c}$ at compressive strength is given and its decomposition into an elastic part $\epsilon_{e d, c}$ and a plastic part $\epsilon_{p, c}$ has to be determined. The subiteration may start with $\epsilon_{p, c}^{1}=0$. The parameters $e_{d}, e_{d 0}$ of the uniaxial stress-strain relation Eq. (59) have to be chosen such that

$$
\mathrm{e}^{-\left(\frac{-\left(\epsilon_{c}-\epsilon_{p, c}^{i}\right)-e_{d 0}}{e_{d}}\right)^{g_{d}}} E\left(\epsilon_{c}-\epsilon_{p, c}^{i}\right)=-f_{c} .
$$

Furthermore, stress should reach its maximum absolute value at $\epsilon_{c}$, i.e. $\mathrm{d} \sigma_{3} / \mathrm{d} \epsilon_{3}=0$ for $\epsilon_{3}=$ $\epsilon_{c}$. A closed solution for $e_{d}, e_{d 0}$ can be found from these conditions. The two plastic material parameters $c_{p}, e_{p}$ remain to be determined. Dilatancy, as it can be observed with concrete, may be used therefore. Volume expansion under uniaxial compression can only be described with plastic deformations, as this is by definition not possible with isotropic damage alone, see Eq. (1) with $\boldsymbol{\epsilon}_{p}=0$. Dilatancy under uniaxial compression is described by Eqs. (66), (67), which provide the plastic longitudinal strain $\epsilon_{p 3}$ and the total volumetric strain $\epsilon_{v o l}$ for a given longitudinal strain $\epsilon_{3}$ or $\epsilon_{e d, 3}$ respectively. The course of $\epsilon_{v o l}$ may to a large extent be controlled by the values of $c_{p}, e_{p}$. As this curve is generally provided as a function of the longitudinal stress, the aforementioned dependency between the sets of damage and plastic material parameters arises. Characteristic points are given by the stress $\sigma_{\text {min }}$ with minimum volume compression and by the stress $\sigma_{\text {turn }}$ on the softening stress branch with zero volume stresses, i.e. with turnover from compaction to dilatancy. These stresses may be solved for longitudinal elastic strains $\epsilon_{e d, 3, \text { min }}$ and $\epsilon_{e d, 3, t u r n}$ by Eq. (59). Each of these values has corresponding plastic strains, which are given by Eq. (66) with 
open values $c_{p}, e_{p}$. Using the pair $\left(\epsilon_{3, t u r n}^{i}, \epsilon_{p, 3, t u r n}^{i}\right)$ with Eq. $(67)$ and the pair $\left(\epsilon_{3, \text { min }}, \epsilon_{p, 3, \text { min }}\right)$ with the derivative of Eq. (67) provides a system of two equations, which can be solved for $c_{p}, e_{p}$.

As all material parameters have been determined in this subiteration loop, a new plastic part $\epsilon_{p, c}^{i+1}$ of the total longitudinal strain $\epsilon_{c}$ can be calculated from Eq. (66). This may serve as a start value for a new subiteration loop. Generally, this subiteration should converge within a few number of loops. It may be embedded in an iteration for the "cross-effect parameter" $c_{c}$ to reach a target value for the remaining plastic longitudinal strains at unloading.

A number of results shall be demonstrated with concrete grades C 20, C 40 and C 60 according to the CEB-modelcode 90 [7]. Some of the basic input parameters like the initial Young's modulus $E$, Poisson's ratio $\nu$, cubic uniaxial compressive strength $f_{c}$, corresponding strain $\epsilon_{c}$ are given by [7]. Other parameters like the two characteristic points of the dilatancy behavior have to be derived from available experimental data, e.g. [24]. The longitudinal plastic strain is not explicitly controlled, as this depends on the point of loading reversal. A value $c_{c}=0.08$ is assumed instead, which leads to reasonable results. The assumed basic material parameters and the derived damage and plastic parameters are given in Table 1. Cubic uniaxial compressive strength has been chosen instead of cylinder uniaxial compressive strength, as this value better matches to experimental data and to values of other codes. Strain values $\epsilon_{c}$ were also slightly increased for C 40 and C 60 for the same reasons. The computed stress-strain relations for uniaxial compression and the stressvolumetric strain relations are shown in Fig. 4 where a homogeneous behavior has been assumed for the hardening as well as for the softening branch. It can be seen, that typical results like dilatancy, loss of stiffness and remaining strains upon unloading are accurately modeled.

So far, only uniaxial compression behavior has been discussed. Tensile and multiaxial behavior is determined by the parameters $b_{1} \ldots b_{4}$, see Eq. (57). With Eqs. (51), (56) these formal parameters can be related to the physical parameters $\alpha_{1}, \alpha_{2}, \alpha_{3}, \beta$, as they have been introduced in connection with failure states for stresses. The parameter $\alpha_{1}$ refers to the uniaxial tensile strength, which 
can be taken from [7]. Computed stress-strain relations for uniaxial tension are given with Fig. 5 where the predefined tensile strengths are exactly met and their strains are good approximations to corresponding values of [7]. This code also provides the data to derive the parameter $\alpha_{2}$ regarding biaxial compressive strength. In contrast, triaxial strength data, as they are necessary for the parameters $\alpha_{3}, \beta$, have to be derived from experimental results, e.g. [19,28,33]. Feasible choices for the parameters $\alpha_{1}, \alpha_{2}, \alpha_{3}, \beta$ are given in Table 2 , but these values are not mandatory and may be varied. Computed results for biaxial strength are shown in Fig. 6 for the selected concrete grades, and include uniaxial tensile strengths and uniaxial compressive strengths as special cases. Basically, the same approach for material parameter determination may be applied to other materials.

\section{Crack Band Model}

The application of the constitutive law to uniaxial tension leads to a limited tensile strength followed by a softening branch in the stress-strain relation, see Fig. 5 . As a well-known fact, this property of the stress-strain behavior leads to localization phenomena and a fundamental mesh sensitivity of numerical calculations. A number of advanced approaches like gradient or nonlocal formulations have been proposed to resolve localization zones and to reach mesh-objective results [2]. These approaches require extensive interventions in finite element implementations. It is furthermore wellknown that more simple approaches like the crack band model may lead to a correctly predicted response on the structural level, while the size of the localization zone spreads over a width of one or two finite elements irrespective of element size.

The crack band model is based on an energy-based rescaling of the softening part of the stressstrain behavior. This will be used for the tension regime of the proposed constitutive law. Tensile failure is characterized by the crack energy

$$
G=\int_{0}^{b_{w}} g(\epsilon) \mathrm{d} b
$$


with the localization zone thickness $b_{w}$, its variable $b$ and the specific crack energy

$$
g(\epsilon)=\int_{\epsilon_{f c t}}^{\epsilon} \sigma_{1}\left(\epsilon^{\prime}\right) \mathrm{d} \epsilon^{\prime}, \quad \epsilon \geq \epsilon_{f c t}
$$

starting from concrete tensile strength with a corresponding strain $\epsilon_{f c t}$ and with $\sigma_{1}(\epsilon)$ according to Eq. (68). The specific crack energy $g$ results from integration of the decreasing branch of the stress-strain relation, see Fig. 5, and hence depends on the other material parameters. Under the assumption that the strain value $\epsilon_{\text {lim }}$ is reached across the whole localization zone, the total crack energy may be approximated by

$$
G_{f} \approx b_{w} g_{f}, \quad g_{f}=g\left(\epsilon_{l i m}\right)
$$

with a strain $\epsilon_{\text {lim }}$ with $\sigma_{1}\left(\epsilon_{\text {lim }}\right) \ll 1$. The total crack energy $G_{f}$ is a material property. Its value is, within a certain range, independent from the other material parameters. This leads to a nominal localization zone width $b_{w}=G_{f} / g_{f}$ as a further characteristic material value, which may be regarded as an internal length scale. Values of $G_{f}$ can again be taken from [7] and are given in Table 3 for the chosen concrete grades where a small aggregate size is assumed with respect to small size structural elements typical for experiments.

In the following, finite element calculations with multiaxial states of stress and strain have to be considered. The basic assumption is that, with respect to crack energy, a multiaxial state with predominant tension can be represented by a uniaxial tension state with the same equivalent damage strain $\kappa_{d}$ defined by Eq. (57). For progressing damage a given value $\kappa_{d}$ also determines the corresponding value $\epsilon_{1}$ of the longitudinal tensile strain for uniaxial loading, see Eq. (70), and the specific crack energy $g_{f}$ for multiaxial states may again be computed from Eqs. (68), (75). Thus, in the following a scaling of $\kappa_{d}$ is used to modify the specific crack energy for arbitrary strain states.

Such a modification may become useful as numerical calculations with finite elements yield a localization zone width corresponding to the width of one or two elements. This width is assumed to be characterized by a value $b_{f e}$, which results in a calculated crack energy

$$
G_{f e} \approx b_{f e} g_{f}
$$


To gain a prescribed crack energy $G_{f}$ with a given element width measure $b_{f e}$ a specific crack energy

$$
g_{f e} \approx \frac{G_{f}}{b_{f e}}=\frac{b_{w}}{b_{f e}} g_{f}
$$

has to be gained on the element level. This ensures mesh objectivity on the structural level as has been discussed before. A variable specific crack energy is reached with a scaled value $\kappa_{d, f e}$ of $\kappa_{d}$. The following three parameter approach is chosen

$$
\kappa_{d, f e}=\gamma_{1}\left(\kappa_{d}-\kappa_{d, l i m}\right)+\frac{1-\gamma_{1}}{\gamma_{2}}\left(1-\mathrm{e}^{-\gamma_{2}\left(\kappa_{d}-\kappa_{d, l i m}\right)}\right)+\kappa_{d, l i m}, \quad \kappa_{d} \geq \kappa_{d, l i m}
$$

with the equivalent strain $\kappa_{d, l i m}$ at the stress limit, see Eq. (45), and two further parameters $\gamma_{1}, \gamma_{2}$. A value $\gamma_{1}=1$ leads to $\kappa_{d, f e}=\kappa_{d}$. Values $\gamma_{1}>1$ lead to an expansion of $\kappa_{d, f e}$ compared to $\kappa_{d}$, while values $\gamma_{1}<1$ lead to a contraction, for a concrete grade C 40 see Fig. 7 . The parameter $\gamma_{2}$ rules the shape of the transition curve from $\kappa_{d}=\kappa_{d, l i m}$ into the area with constant slope of $\kappa_{d, f e}$. A fixed value of $\gamma_{2}=350$ has proved to be adequate. Such a value $\kappa_{d, f e}$, defined by Eq. (79), can replace $\kappa_{d}$ in Eqs. (70), (68) and (75) to compute the specific crack energy $g_{\gamma}\left(\gamma_{1}\right)$ with a variable parameter $\gamma_{1}$. A closed-form solution of these equations is shown in Fig. 8 for a concrete grade C 40 where the ratio $g_{\gamma} / g_{f}$ is given depending on $\gamma_{1}$. With a target value $g_{f e}$ of the specific crack energy given by Eq. (78), a value of the scaling parameter $\gamma_{1}$ is derived from Fig. 8 . The effects of damage scaling become especially evident for the homogeneous uniaxial tension case, see Eq. (68). Inserting Eq. (70) into (79), while the latter replaces $\kappa_{d}$ in Eq. (68), results in a stress-strain relation parametrized by $\gamma_{1}$. This is demonstrated in Fig. 9 for the concrete grade $\mathrm{C} 40$. It can be seen that values $\gamma_{1}>1$ cause a shortening of the stress-strain relation in the softening range. In contrast, values $\gamma_{1}<1$ cause an extension, while the tensile strength remains unchanged. Summarizing, a crack energy value $G_{f}$ is ensured for softening elements of varying size, at least for Mode-I conditions and element edges aligned to the tensile direction. This approach requires a minimum effort while preserving a consistent tangent material stiffness.

Some margin of estimation remains with the selection of $b_{f e}$ for softening elements. Generally, nearly quadratic elements are striven to improve the interpolation quality. Thus, a natural choice 
for $b_{f e}$ is given by an element's edge length. On the other hand numerical calculations show that softening may spread over two elements normal to the localization direction if the localization is not aligned with mesh orientations. Therefore, in the following numerical calculation $b_{f e}$ is generally chosen 1.5 times the mean of the element's edge length in case of softening elements. Furthermore, questions remain with Mixed-Mode conditions. The Finite Element Method allows to distinguish Mode-I and Mode-II contributions to the fracture energy and to compute their values for a whole system using the integration point values. Taking also crack band geometries into consideration this leads to overall correct crack energy values, as it is also confirmed by the computed loaddisplacement relations in the softening range, see the following section.

As an alternative to a crack band regularization a gradient damage approach has been investigated with the proposed constitutive law where a nonlocal field of the equivalent damage strain $\kappa_{d}$ was introduced as further global independent variable [15]. This basically gives the same results for the global system behavior, but proves to be more expensive and less robust from a computational point of view in the investigated cases. More detailed investigations regarding optimization of regularization methods have to be done in future work.

\section{Applications}

Only uniaxial compression and tension have been discussed so far, which were assumed to be homogeneous and so could be realized with one-element models. Practical applications show nonhomogeneous behavior and require a large number of elements of different size and varying shape. Furthermore, reinforcement and bond between reinforcement and concrete play a role. This creates its own issues, which will not be covered within this paper. Thus, the following application examples are restricted to plain concrete structures without reinforcement. The behavior of such structures is generally dominated by tension failure of concrete. The L-shaped panel is studied as an example for Mode-I failure and the four-point shear beam as an example for Mixed-Mode failure. Plane 
stress conditions are assumed in all cases. The structure's loading is controlled by prescribing the displacement of particular nodes. The Newton-Raphson method combined with an arc-length control of loading steps is used for the equilibrium iterations. A band of high damage can be seen in Fig. 16 for the central area around the notch in a gray scale view on shrunken elements. The L-shaped panel has become a popular benchmark test for the validation of computational models of plain concrete under Mode-I conditions, i.e. with cracking caused by predominant tensile stresses. It was experimentally investigated by [39]. The geometry and the boundary conditions are shown in Fig. 10. The available parameters of the material used in the experiments are listed in Table 4. Remarkable is the low Young's modulus compared to the strength. As it is not intended to perform a parameter fitting study, the concrete grades C 20 and C 40 according to Tables $1-3$ are chosen for a comparison without modifications, because they prove to be limiting cases. The finite element discretization, which is indicated with a shrunken element view in Fig. 12, has 2161 nodes and 2079 four node quadrilaterals. The computed load-displacement relations together with the scatter of the experimental data are given in Fig. 11. The initial stiffness of the experimental panels falls sightly below the computational results due to the low experimental Young's modulus. All cases have the same characteristics with a limit load followed by descending branches. A descending branch is connected with localizations in the numerical calculations, which can be seen in Fig. 12 with the computed scalar damage for the case of concrete grade C 40 in the last computed step. The damage value is indicated by a gray scale upon a shrunken element view. A concentration of damage with values approaching 1 develops in a horizontally banded area. This indicates a horizontal crack direction, which is typical for other isotropic numerical models [11]. The experimentally observed macro-cracking area actually shows a slight inclination in the upper direction. Anisotropic damage models are presumably required to describe this behavior. Computed principal stresses of a central section are given in Fig. 13 with high concentrated compressive stresses in the narrow part left to the crack tip area and tensile stresses right to the crack tip area, which decrease with increasing 
element strains or crack widths respectively.

Experiments with the four-point shear beam with a notch were performed by [1] to study the behavior of plain concrete under Mixed-Mode conditions, which combine shear stresses and normal stresses along the cracking planes. Geometry and boundary conditions are shown in Fig. 14. The experimental material parameters are given in Table 4 as far as they are known. Again, a quite low Young's modulus can be seen in relation to the strength. Treating this system as a beam, it has a high shear force combined with a nearly vanishing bending moment in a cross section coinciding with the notch. In a strut-and-tie model, a line of high compression develops between the upper and lower bearing points $A$ and $B$, see Fig. 14. All three concrete grades, which have been discussed in the foregoing Section 5, are used for a comparison. The finite element discretization has 4837 nodes and 4662 four node quadrilaterals. The central part of it is indicated in Fig. 16 in a shrunken element view. The upper bar for load distribution is assumed with a linear elastic material of very high stiffness, so it behaves rigid.

Deformations due to cracking are indicated by the crack mouth opening displacement (cmod), which is the difference of the vertical displacements of points $C$ and $D$, see Fig. 14 . The loadcmod curves for the different cases are shown in Fig. 15. Generally, no equilibrium is found shortly after reaching the peak loads in the numerical computations. Hence, a distinct softening behavior could not be computed. Regarding the peak, it can be seen that the corresponding displacements are underestimated by the numerical calculations by about $15 \%$ while the peak load has the best agreement with a concrete C 40, which also agrees best with the compressive strength. A band of high damage can be seen in Fig. 16 for the central area around the notch in a gray scale view on shrunken elements. It occurs for the concrete grade C 40 at the end of the computation and has traversed the beam height to a large extent. Similar to the L-shaped panel, the concentrated damage band shows more or less straight segments while the actually observed macro-cracks are curved from the notch tip towards the point $A$. Again, an anisotropic damage model and a finer 
finite element resolution seems to be necessary to model this behavior. Analyzing principal stresses at integration points in their peak load state, i.e. with $\kappa_{d}=\kappa_{d, l i m}$, a Rankine-like state with principal stresses $\sigma_{1} \approx f_{c t},\left|\sigma_{2}\right| \ll\left|\sigma_{1}\right|$ is given at the beginning of crack propagation, while this later changes to pure shear with $\sigma_{1} \approx-\sigma_{2} \approx f_{c t}$ when progressing to the upper bearing point A. Computed principal stresses for concrete grade C 40 in the central beam section are shown in Fig. 17 for the peak load state. This shows a compressive arch adjacent to the high damage zone, which transfers loads between the upper and lower bearing points $A, B$. Stresses are sharply reduced below this compressive arch. Altogether, it can be seen that a sharp, load-induced change in stresses and material state can be modeled with the proposed constitutive law in combination with the crack band approach.

\section{Conclusions and Outlook}

A three-dimensional constitutive law for concrete has been proposed, based on isotropic damage combined with plasticity. A key point is given with the transfer of strength failure criteria from stress space into strain space and parametrizing the strain-based formulation with an equivalent strain. This equivalent damage strain is coupled to a measure for isotropic damage. This is combined with an approach for plastic strains to cover effects like remaining strains upon unloading and dilatancy. With such a strain-based approach, relatively simple forms compared to stress-based approaches are derived for the formulation of the constitutive law, the corresponding numerical algorithms and its regularization. Nevertheless, typical characteristics of concrete behavior are reproduced for a wide range of concrete grades and the behavior of major benchmark problems for plain concrete are generally correctly modeled.

Extensions of this constitutive law may be discussed under two major aspects. First of all, anisotropic damage may be regarded. Out of this, orthotropic damage may be realized using the proposed isotropic law by transferring its formulation to each direction within the framework set 
by [4]. This is especially relevant for load-induced anisotropy and should lead to a more exact prediction of paths of localization zones or macrocracks respectively. On the other hand, the effort of anisotropic formulations strongly grows and for practical applications, it has to be assessed whether the expense is worthwhile compared to the modeling benefit.

A further aspect concerns regularization. Enhanced methods like gradient continuum models are necessary to resolve the bandwidth of localization zones and avoid modifications of the constitutive law depending on measures of finite element size. In the present context, this may be performed with the introduction of an averaged equivalent damage strain, which is ruled by an additional differential equation or its weak formulation respectively. However, again the effort grows and additional length scales have to be introduced as material parameters, which have to be calibrated against observed material behavior. An alternative to circumvent mesh sensitivity of continuous displacement approaches is given by using discontinuous approaches like XFEM or element free Galerkin methods to model crack propagation in combination with traction-separation laws. But this creates own issues like criteria for the transition from continuum to discontinuum and appropriate traction-separation laws, e.g. laws for Mixed-Mode conditions.

A basic problem common to all methods and approaches concerns the availability of material property data for concrete and the scatter of this data. Often the required data to determine the parameters for advanced constitutive laws are not available under practical application conditions. Thus, one has to deal with basic parameters like the initial Young's modulus, initial Poisson's ratio and the uniaxial compressive and tensile strength and has to derive advanced parameters on base of plausibility and general experience. This requires studies of the sensitivity of computational results on variations of advanced parameters and remains to be investigated for the constitutive law proposed here. Concerning the given scatter of all material properties, this problem may be generalized with sensitivity studies regarding all material parameters and may be extended to probabilistic numerical methods or other random approaches. 


\section{Notation}

The following symbols are used in this paper:

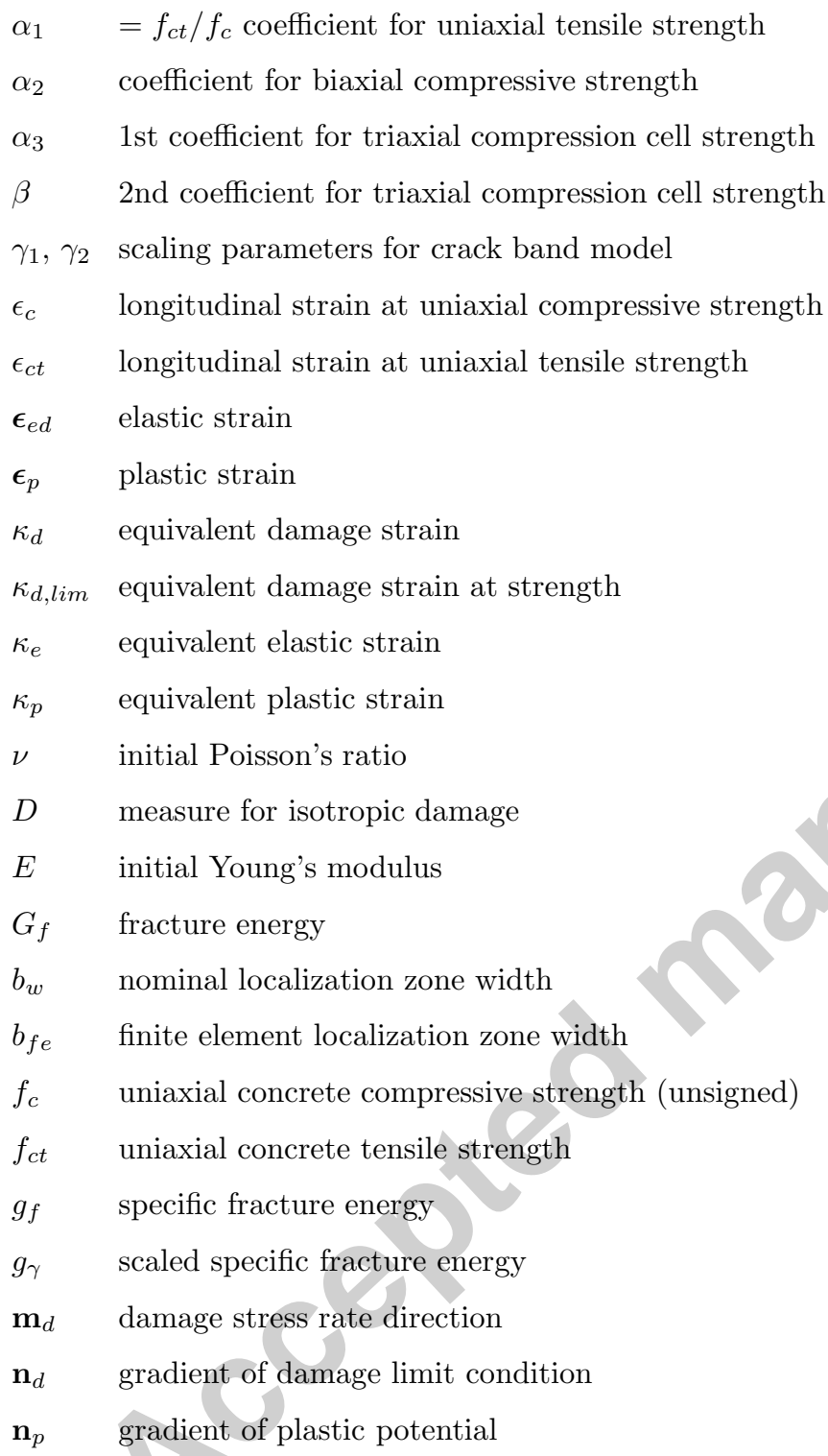




\section{References}

[1] M. Arrea and A.R. Ingraffea. Mixed-mode crack propagation in mortar and concrete. Technical Report 81-13, Dep. of Struct. Eng., Cornell University, Ithaka, 1982.

[2] Z.P Bažant and M. Jirázek. Nonlocal integral formulations of plasticity and damage: Survey of progress. Journal of Engineering Mechanics, 128:1119-1149, 2002.

[3] I. Carol, E. Rizzi and K. Willam. A unified theory of elastic degradation and damage based on a loading surface. Int. J. Solids Structures, 31:2835-2865, 1994.

[4] I. Carol, E. Rizzi and K. Willam. On the formulation of anisotropic elastic degradation. I. Theory based on a pseudo-logarithmic damage tensor rate. II. Generalized pseudo-Rankine model for tensile damage. Int. J. Solids Structures, 38:491 - 546, 2001.

[5] W.F. Chen and A.F. Saleeb. Constitutive Equations for Engineering Materials, Volume 1: Elasticity and Modeling. Elsevier Science B.V., Amsterdam, 2nd edition, 1994.

[6] W.F. Chen and A.F. Saleeb. Constitutive Equations for Engineering Materials, Volume 2: Plasticity and Modeling. Elsevier Science B.V., Amsterdam, 1994.

[7] Comité Euro-International du Béton. CEB-FIP Model Code 1990. Thomas Telford, London, 1993.

[8] M. Curbach, T. Hampel, S. Scheerer and K. Speck. Hochleistungsbeton unter zwei- und dreiaxialer Beanspruchung. Beton- und Stahlbetonbau, 97:275-280, 2002.

[9] J.W. Dougill. On stable progressively fracturing solids. J. Appl. Math. Phys., 27:423-437, 1976.

[10] G. Etse and K. Willam. Fracture energy formulation for inelastic behavior of plain concrete. Journal of Engineering Mechanics, 120:1983-2011, 1994.

[11] Ch. Feist, W. Kerber, H. Lehar and G. Hofstetter. A comparative study of numerical models for concrete cracking. In: P. Neittaanmäki, et al.: Proceedings of European Congress on Computational Methods in Applied Sciences and Engineering (ECCOMAS 2004) (CD-ROM), 2004.

[12] K.H. Gerstle, et al. Behavior of concrete under multiaxial stress states. Journal of Engineering Mechanics, 106:1383-1403, 1980.

[13] S. Govindjee, G.J. Kay and J.C. Simo. Anisotropic modelling and numerical simulation of brittle damage in concrete. International Journal for Numerical Methods in Engineering, 38:3611$3633,1995$.

[14] Z. Guo, Y. Zhou and D. Nechvatal. Evaluation of the Multiaxial Strength of Concrete Testet at the Technische Universität München. Deutscher Ausschuss für Stahlbeton (DAfStb) Heft 447, 1995. 
[15] U. Häußler-Combe. Zur Verwendung von Stoffgesetzen mit Entfestigung in numerischen Rechenverfahren. Bauingenieur, 82:286-298, 2007.

[16] E. Hansen, K. Willam and I. Carol. A two-surface anisotropic damage/plasticity model for plain concrete. In R. de Borst et al., editor, Fracture Mechanics of Concrete Structures, pp. 549-556. Swets \& Zeitlinger, Lisse, 2001.

[17] S.S. Hsieh, E. Ting and W.F. Chen. A plasticity fracture-model for concrete. Int. J. Solids Structures, 18:181-197, 1982.

[18] A. Hussein and H. Marzouk. Behavior of high-strength concrete under biaxial stresses. ACIMaterials Journal, 97:27-37, 2000.

[19] I. Imran and J. Pantazopoulou. Experimental study of plain concrete under triaxial stress. ACI Materials Journal, 93:589-601, 1996.

[20] M. Jirázek and Z.P. Bažant. Inelastic Analysis of Structures. John Wiley \& Sons, New York, 1st edition, 2002.

[21] L.M. Kachanov. On rupture time under condition of creep. In Otd. Techn. Nauk, 8:26-31. Izvestia Akademi Nauk USSR, 1958.

[22] M.D. Kotsovos and J.B. Newman. Generalized stress-strain relations for concrete. Journal of Engineering Mechanics, 104:845-856, 1978.

[23] W. Krätzig and J. Pölling. An elasto-plastic damage model for reinforced concrete with a minimum number of material parameters. Computers \& Structures, 82:1201-1215, 2004.

[24] H. Kupfer and H.K. Hilsdorf. Behavior of concrete under biaxial stresses. ACI Journal, 66:656$666,1969$.

[25] J. Lemaitre. Coupled elasto-plasticity and damage constitutive equations. Comp. Meth. Appl. Mech. Eng., 51:31-49, 1985.

[26] J. Lemaitre. A Course on Damage Mechanics. Springer-Verlag, Berlin, 1992.

[27] J. Lemaitre and R. Desmorat. Engineering Damage Mechanics. Springer-Verlag, Berlin, 2005.

[28] Q. Li and F. Ansari. Mechanics of damage and constitutive relationships for high-strength concrete in triaxial compression. Journal of Engineering Mechanics, 125:1-10, 1999.

[29] J. Mazars and G. Pijaudier-Cabot. Continuum damage theory - application to concrete. Journal of Engineering Mechanics, 115:345-365, 1989.

[30] M. Ortiz. A constitutive theory for the inelastic behavior of concrete. Mechanics of Materials, 4:67-93, 1985.

[31] N.S. Ottosen. A failure criterion for concrete. Journal of Engineering Mechanics, 103:527-535, 1977. 
[32] E. Rizzi, G. Maier and K. Willam. On failure indicators in multi-dissipative materials. Int. J. Solids Structures, 33:3187-3214, 1996.

[33] D. Sfer, I. Carol, R. Gettu, and G. Etse. Study of the behavior of concrete under triaxial compression. Journal of Engineering Mechanics, 128:156-163, 2002.

[34] J.C. Simo and T.J.R. Hughes. Computational Inelasticity. Springer-Verlag, New York, 1st edition, 1998.

[35] J.C. Simo and J.W. Ju. Strain- and stress-based continuum damage models - I. Formulation, II. Computational aspects. Int. J. Solids Structures, 23:821-869, 1987.

[36] J.G.M. van Mier. Fracture of concrete under complex stress. Heron, 31: No. 3, 1-90, 1986.

[37] C. Wang, Z. Guo, and X. Zhang. Experimental investigation of biaxial and triaxial compressive concrete strength. ACI Materials Journal, 84:92-100, 1987.

[38] K.J. Willam and E. Warnke. Constitutive model for the triaxial behavior of concrete. In IABSE Proceedings Vol. 19. International Association for Bridge and Structural Engineering, 1975 .

[39] B. Winkler. Traglastuntersuchungen von unbewehrten und bewehrten Betonstrukturen auf der Grundlage eines objektiven Werkstoffgesetzes für Beton. PhD thesis, University of Innsbruck, 2001. 


\section{Tables}

Table 1: Material parameters for uniaxial compression

\begin{tabular}{lcccc}
\hline concrete grade & & $\mathrm{C} 20$ & $\mathrm{C} 40$ & $\mathrm{C} 60$ \\
\hline Young's modulus $E$ & $\mathrm{MN} / \mathrm{m}^{2}$ & 30000 & 36000 & 41000 \\
Poisson's ratio $\nu$ & - & 0.20 & 0.20 & 0.20 \\
cubic compressive strength $f_{c}$ & $\mathrm{MN} / \mathrm{m}^{2}$ & 25 & 50 & 70 \\
strain $\epsilon_{c}$ at compressive strength & - & $-2.2 \cdot 10^{-3}$ & $-2.5 \cdot 10^{-3}$ & $-2.7 \cdot 10^{-3}$ \\
\hline plastic threshold & - & $0.55 \cdot \epsilon_{c}$ & $0.6 \cdot \epsilon_{c}$ & \\
stress $\sigma_{\text {min }}$ with minimum volume strain & $\mathrm{MN} / \mathrm{m}^{2}$ & $-0.92 \cdot f_{c}$ & $-0.92 \cdot f_{c}$ & $-0.92 \cdot f_{c}$ \\
stress $\sigma_{\text {turn }}$ with volume strain turnover & $\mathrm{MN} / \mathrm{m}^{2}$ & $-0.96 \cdot f_{c}$ & $-0.96 \cdot f_{c}$ & $-0.96 \cdot f_{c}$ \\
\hline cross-effect parameter $c_{c}$ & - & 0.08 & 0.08 & 0.08 \\
damage exponent $g_{d}$ & - & 2.0 & 2.0 & 2.0 \\
damage parameter $e_{d 0}$ & - & $-1.54 \cdot 10^{-3}$ & $-6.77 \cdot 10^{-6}$ & $6.58 \cdot 10^{-4}$ \\
damage parameter $e_{d}$ & - & $3.79 \cdot 10^{-3}$ & $3.25 \cdot 10^{-3}$ & $2.98 \cdot 10^{-3}$ \\
plastic parameter $c_{p}$ & - & 3.32 & 3.69 & 3.56 \\
plastic parameter $e_{p 0}$ & - & $4.84 \cdot 10^{-4}$ & $6.00 \cdot 10^{-4}$ & $7.02 \cdot 10^{-4}$ \\
plastic parameter $e_{p}$ & - & $3.15 \cdot 10^{-4}$ & $3.02 \cdot 10^{-4}$ & $1.41 \cdot 10^{-4}$ \\
\hline
\end{tabular}


Table 2: Material parameters for uniaxial tension and multiaxial behavior

\begin{tabular}{lcccc}
\hline concrete grade & & C20 & C40 & C60 \\
\hline tensile strength $f_{c t}$ & $\mathrm{MN} / \mathrm{m}^{2}$ & 2.2 & 3.5 & 4.6 \\
uniaxial tension $\alpha_{1}$ & - & 0.088 & 0.070 & 0.066 \\
biaxial compression $\alpha_{2}$ & - & 1.24 & 1.12 & 1.09 \\
triaxial compression $\alpha_{3}$ & - & 2.0 & 2.0 & 2.0 \\
confining pressure $\beta$ & - & 0.2 & 0.2 & 0.2 \\
\hline limit condition parameter $b_{1}$ & - & 2.2587 & 3.1819 & 3.4522 \\
limit condition parameter $b_{2}$ & - & 0.5334 & -0.3419 & -0.6140 \\
limit condition parameter $b_{3}$ & - & 8.7041 & 11.7710 & 12.6965 \\
limit condition parameter $b_{4}$ & - & 3.6576 & 4.4077 & 4.6183 \\
\hline
\end{tabular}


Table 3: Material parameters for fracture

\begin{tabular}{lcccc}
\hline concrete grade & & C20 & C40 & C60 \\
\hline fracture energy $G_{f}$ & $\mathrm{Nm} / \mathrm{m}^{2}$ & 50 & 70 & 95 \\
specific fracture energy $g_{f}$ & $\mathrm{MNm} / \mathrm{m}^{3}$ & 467.4 & 562.4 & 671.9 \\
nominal localization zone width $b_{w}$ & $\mathrm{~m}$ & 0.1069 & 0.1244 & 0.1413 \\
\hline
\end{tabular}


Table 4: Material parameters of selected experiments

\begin{tabular}{lccc}
\hline experiment & & L-shaped panel & $\begin{array}{c}\text { notched beam } \\
\text { series B }\end{array}$ \\
\hline Young's modulus $E$ & $\mathrm{MNm} / \mathrm{m}^{3}$ & 25850 & 24800 \\
Poisson's ratio $\nu$ & - & 0.18 & 0.18 \\
compressive strength $f_{c}$ & $\mathrm{MNm} / \mathrm{m}^{3}$ & 31 & 45.5 \\
tensile strength $f_{c t}$ & $\mathrm{MNm} / \mathrm{m}^{3}$ & 2.7 & - \\
fracture Energy $G_{f}$ & $\mathrm{Nm} / \mathrm{m}^{2}$ & 90 & - \\
\hline
\end{tabular}




\section{Figures}

Figure 1: Damage $D$ depending on damage equivalent strain $\kappa_{d}$

Figure 2: Plastic equivalent strain $\kappa_{p}$ depending on elastic equivalent strain $\kappa_{e}$

Figure 3: Failure surface Eq. (46) with a view from the negative hydrostatic axis

Figure 4: Stress-strain behavior under uniaxial compression

Figure 5: Stress-strain behavior under uniaxial tension

Figure 6: Biaxial strength

Figure 7: Scaled equivalent damage strain $\kappa_{d, f e}$ for C 40

Figure 8: Specific crack energy gain for C 40 depending on scaling factor $\gamma_{1}$

Figure 9: Uniaxial tension for C 40 with scaled equivalent damage strain $\kappa_{d, f e}$

Figure 10: L-shaped panel: geometry and boundary conditions

Figure 11: L-shaped panel: load-displacement curves

Figure 12: L-shaped panel: damage $D$ for C 40

Figure 13: Central part of L-shaped panel: principal stresses for C 40

Figure 14: Four-point shear beam: geometry and boundary conditions

Figure 15: Four-point shear beam: load-cmod curves

Figure 16: Central part of Four-point shear beam: damage $D$ for C 40

Figure 17: Central part of Four-point shear beam: principal stresses for C 40 


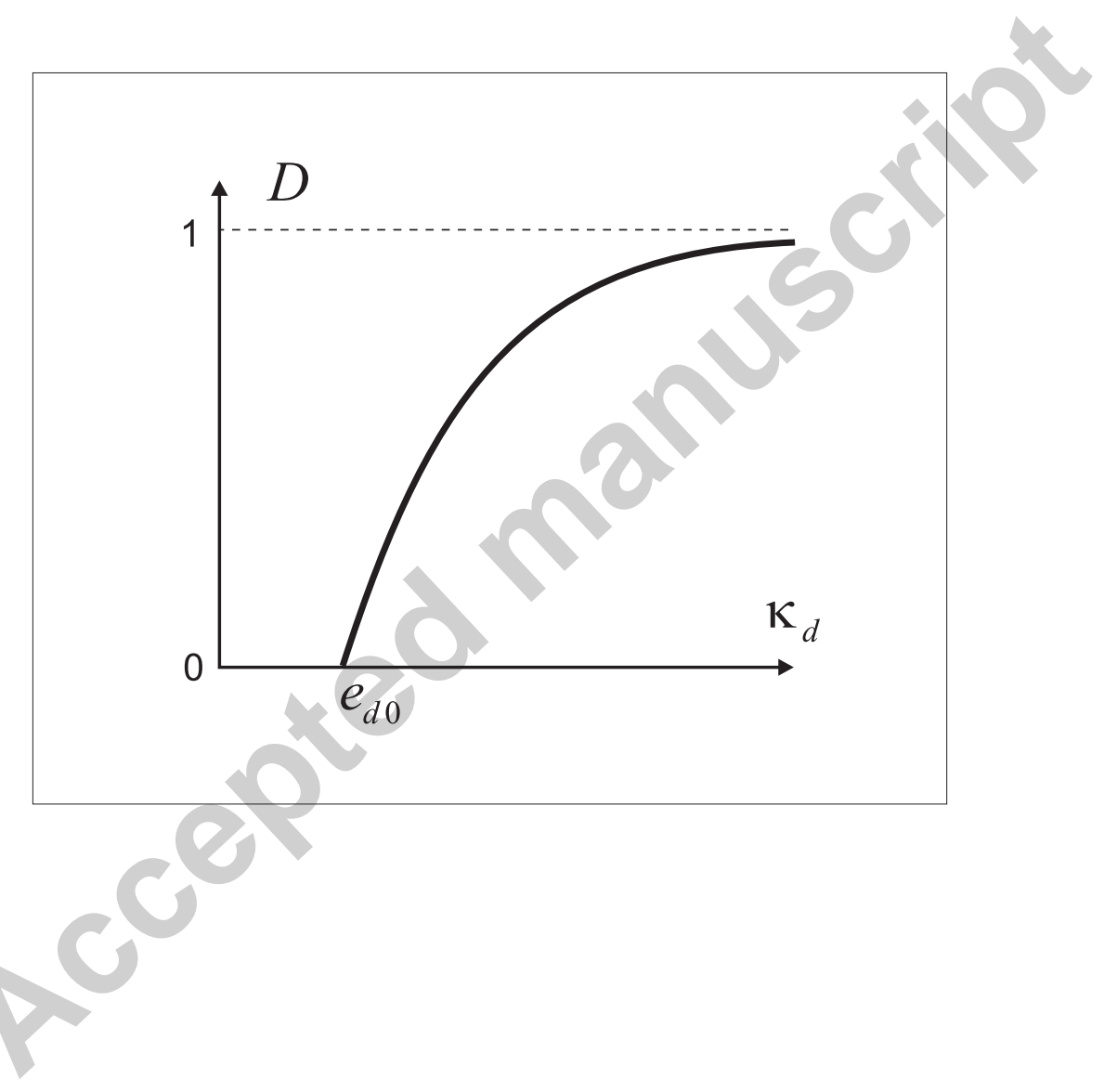




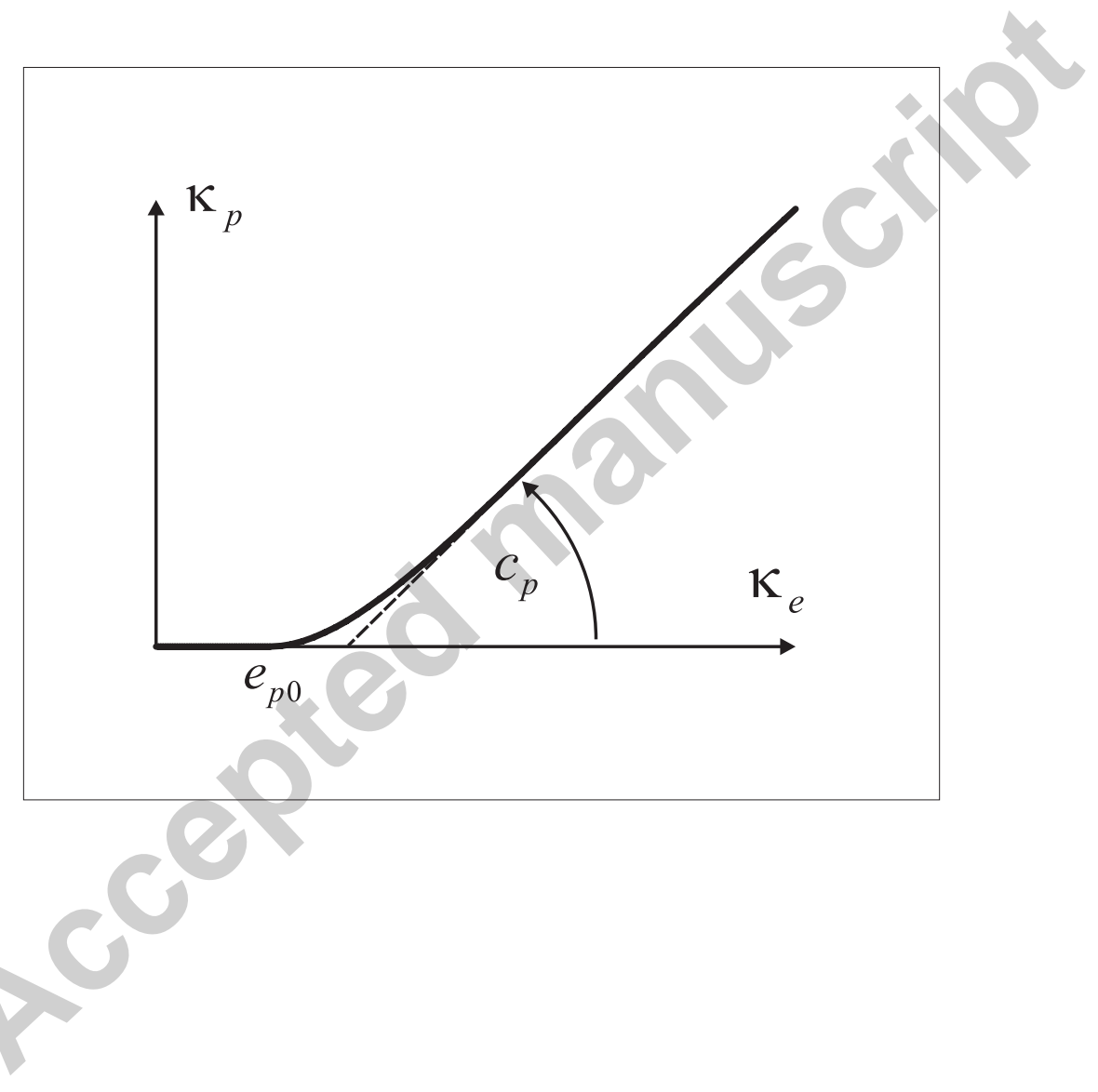

Figure 


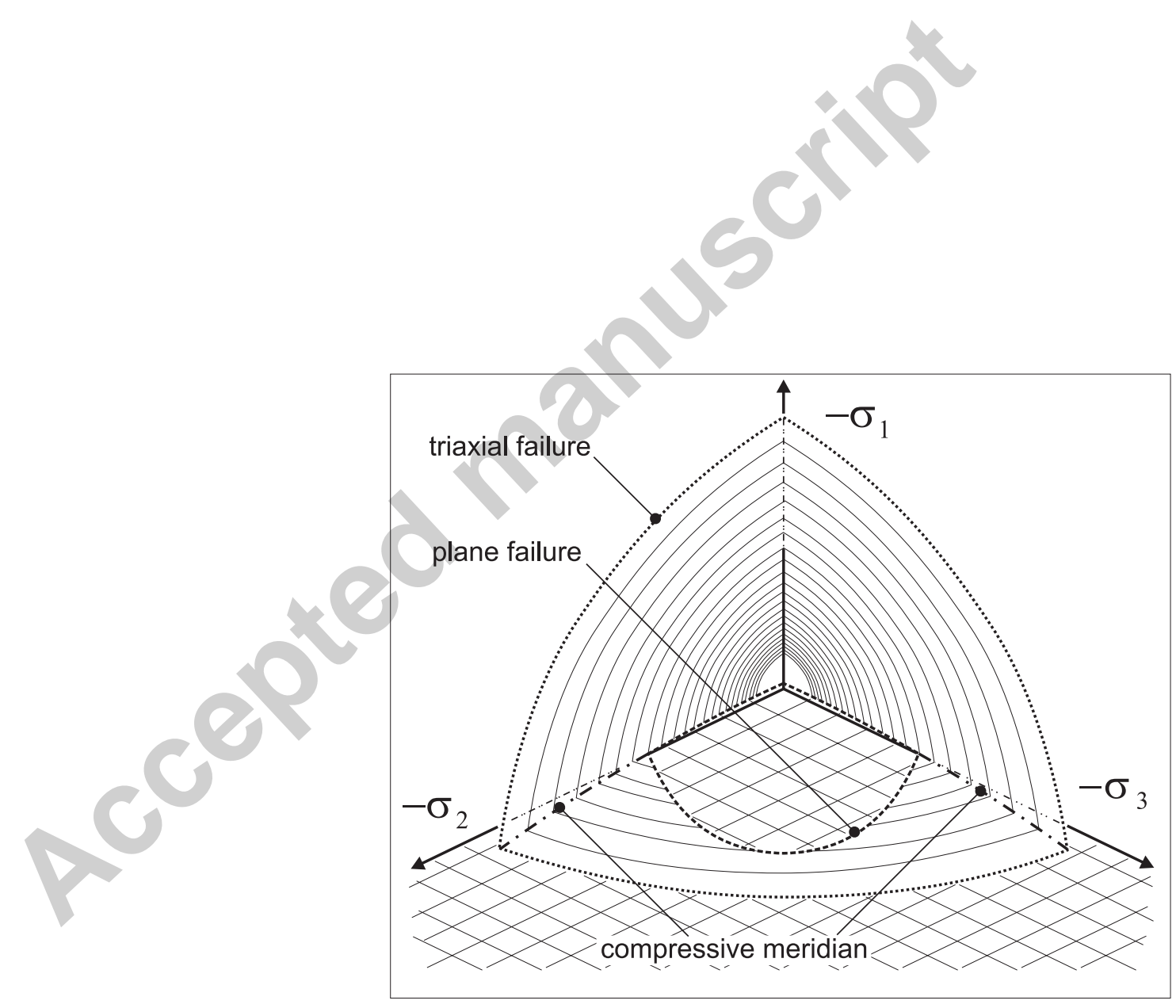

Figure 3 
Figure 4

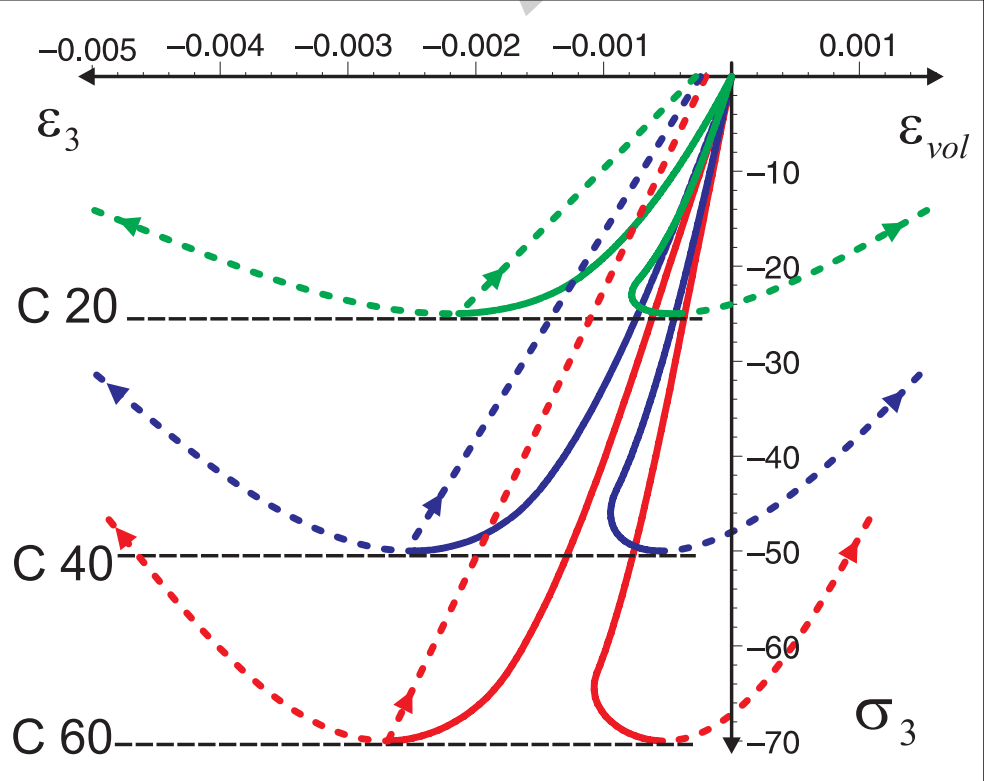


Figure 6

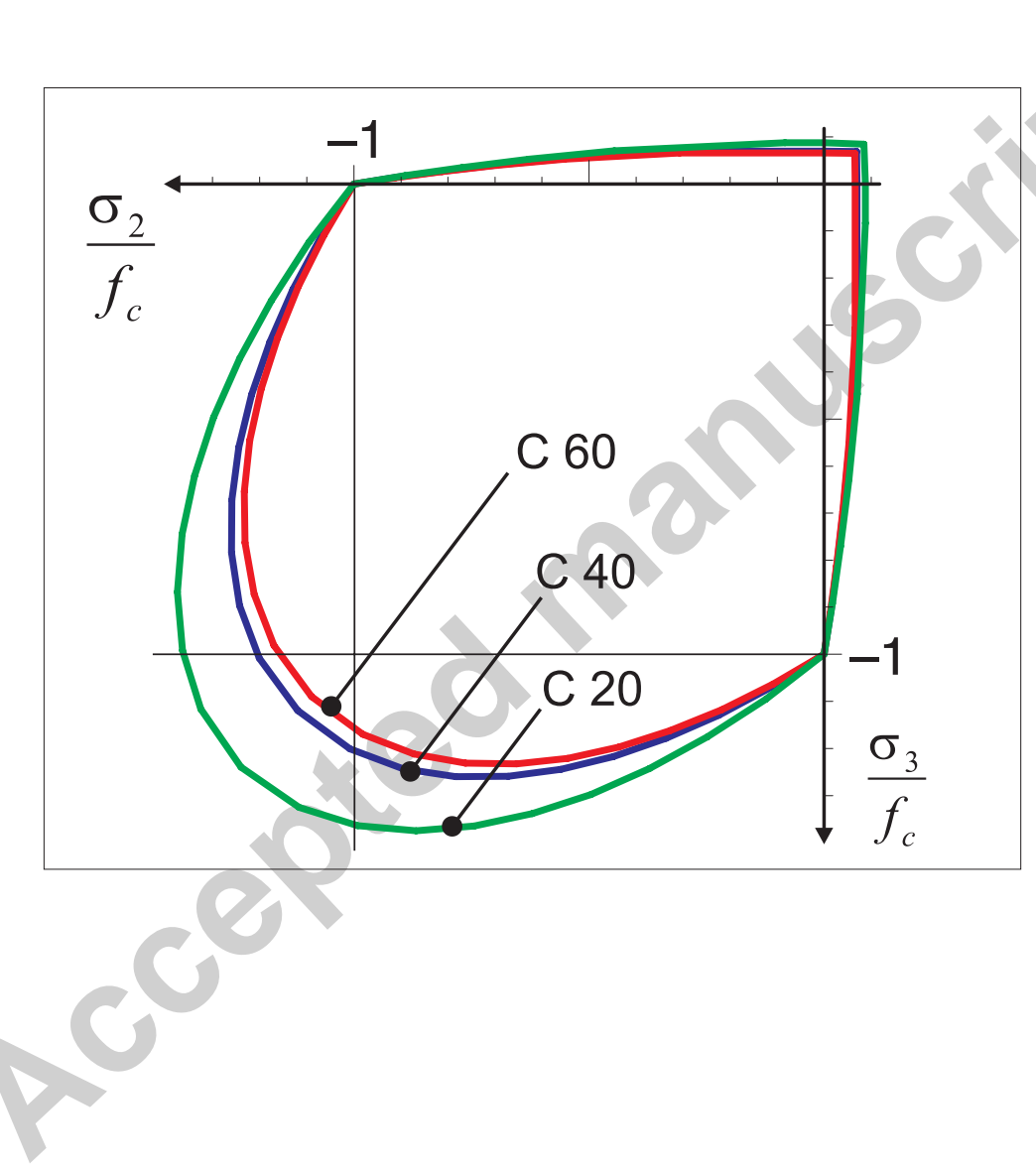




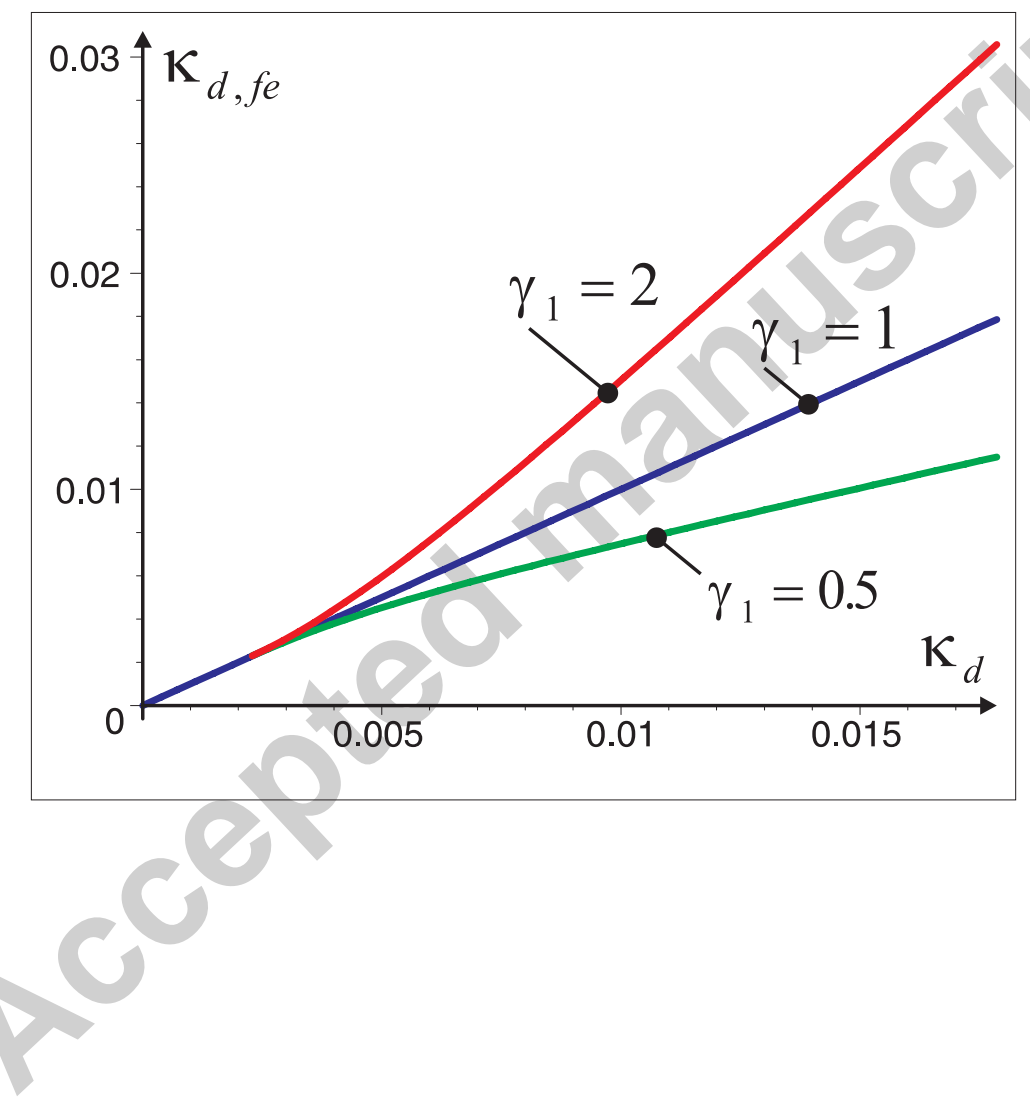


Figure 8

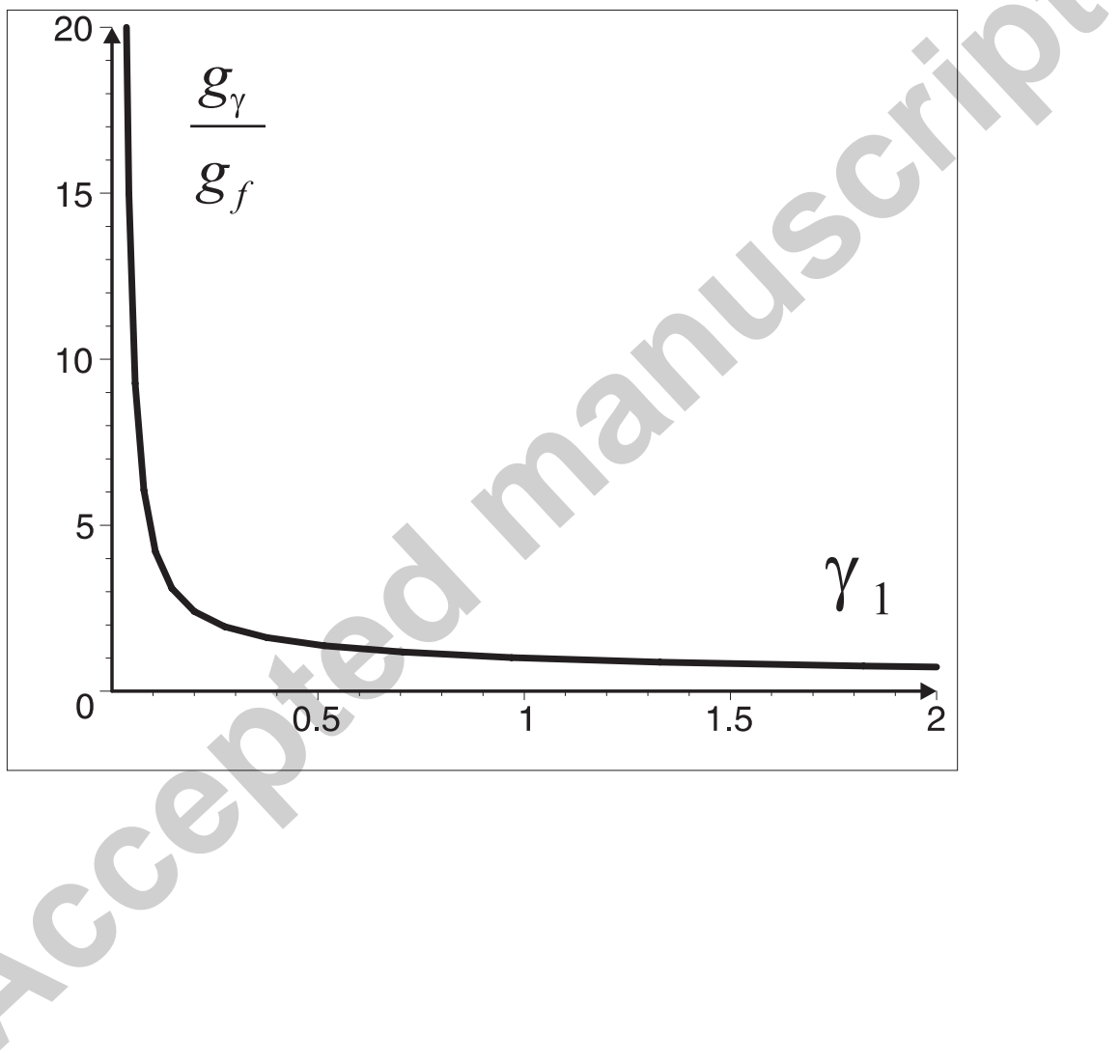


Figure 9

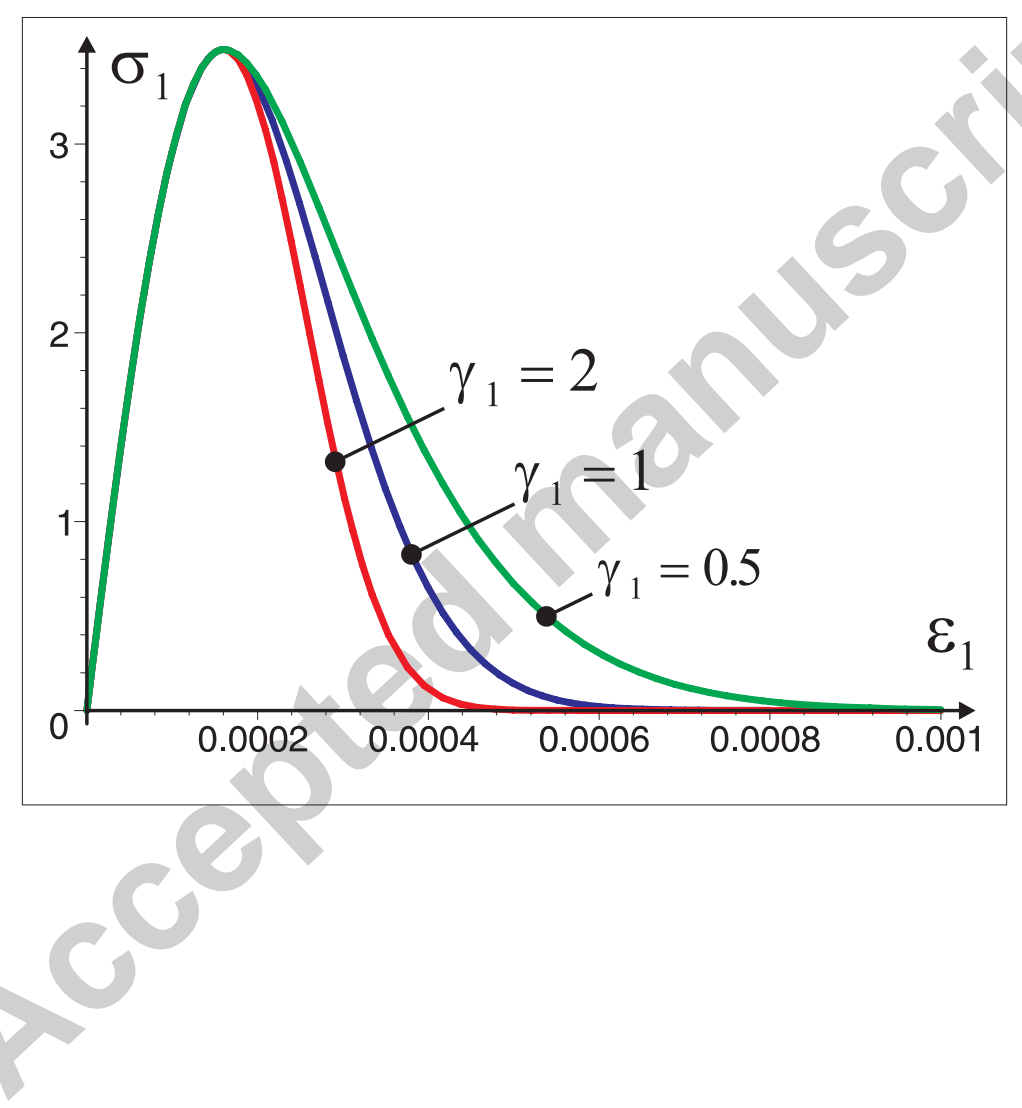




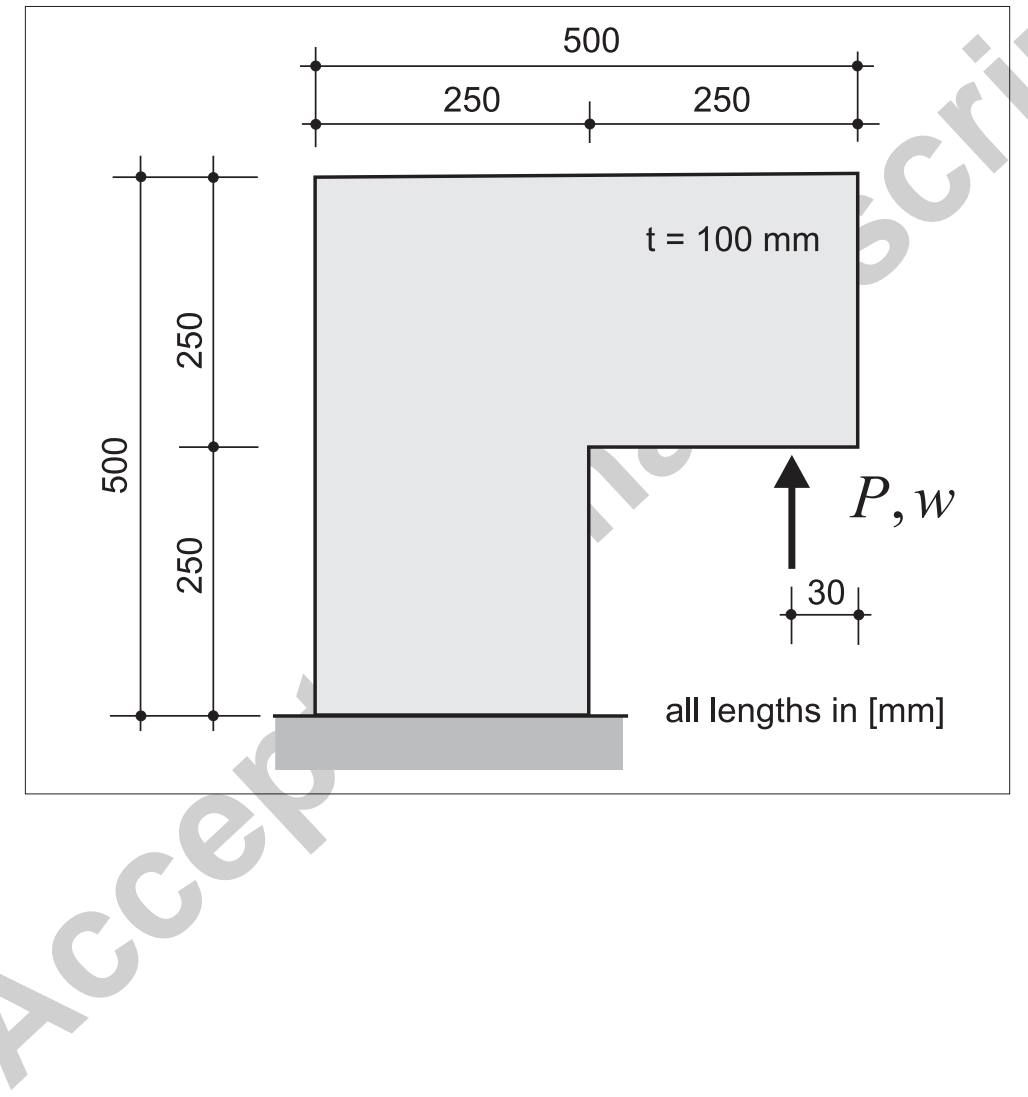




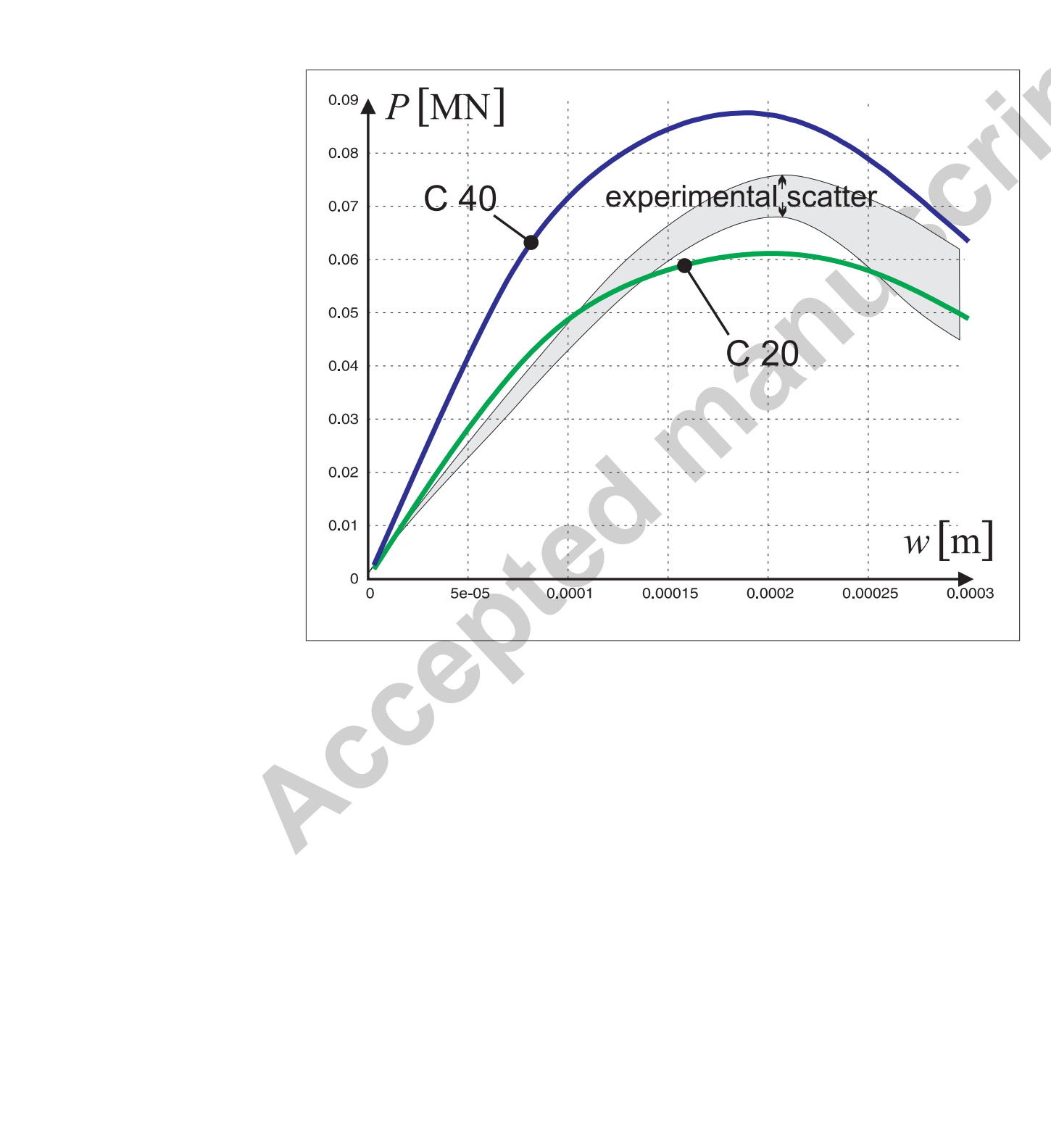

Figure 11

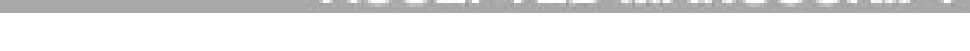

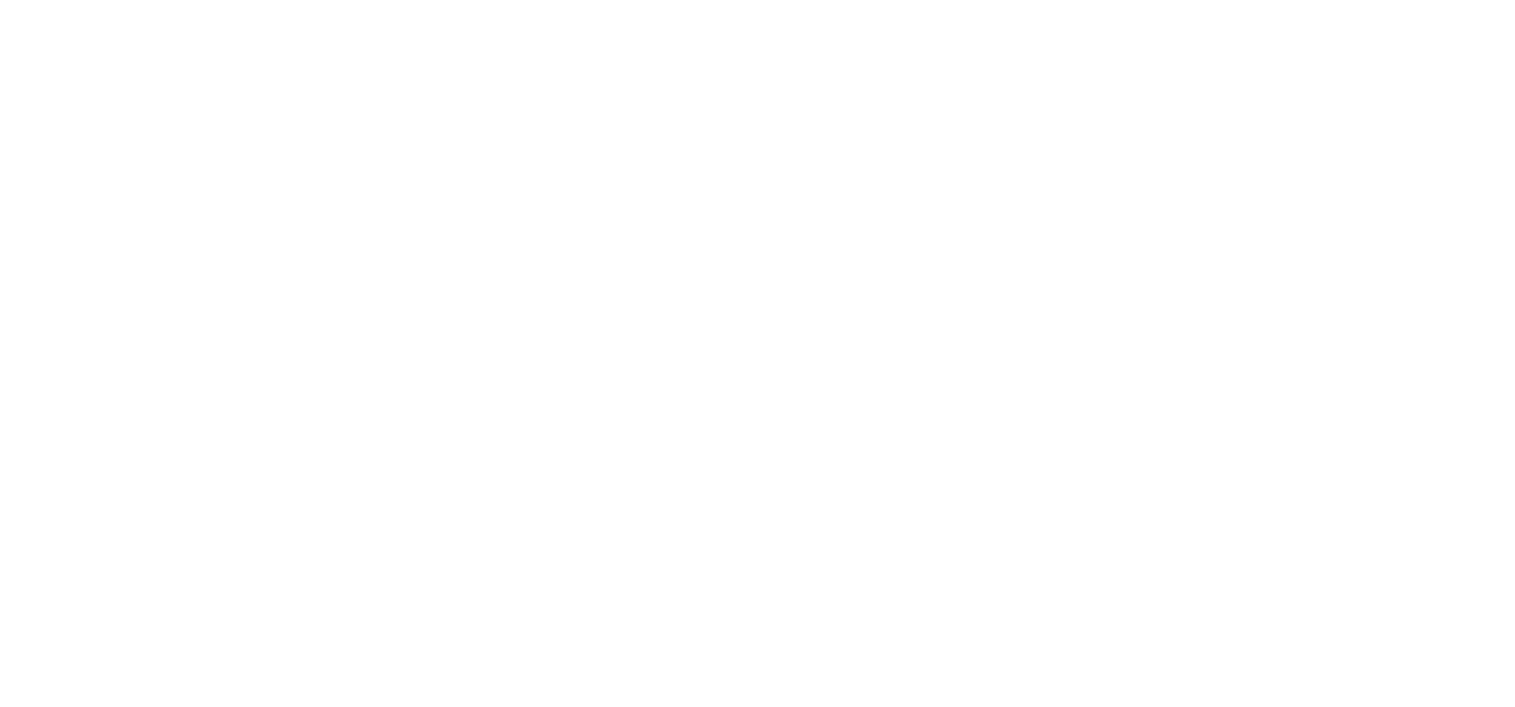

(1) 


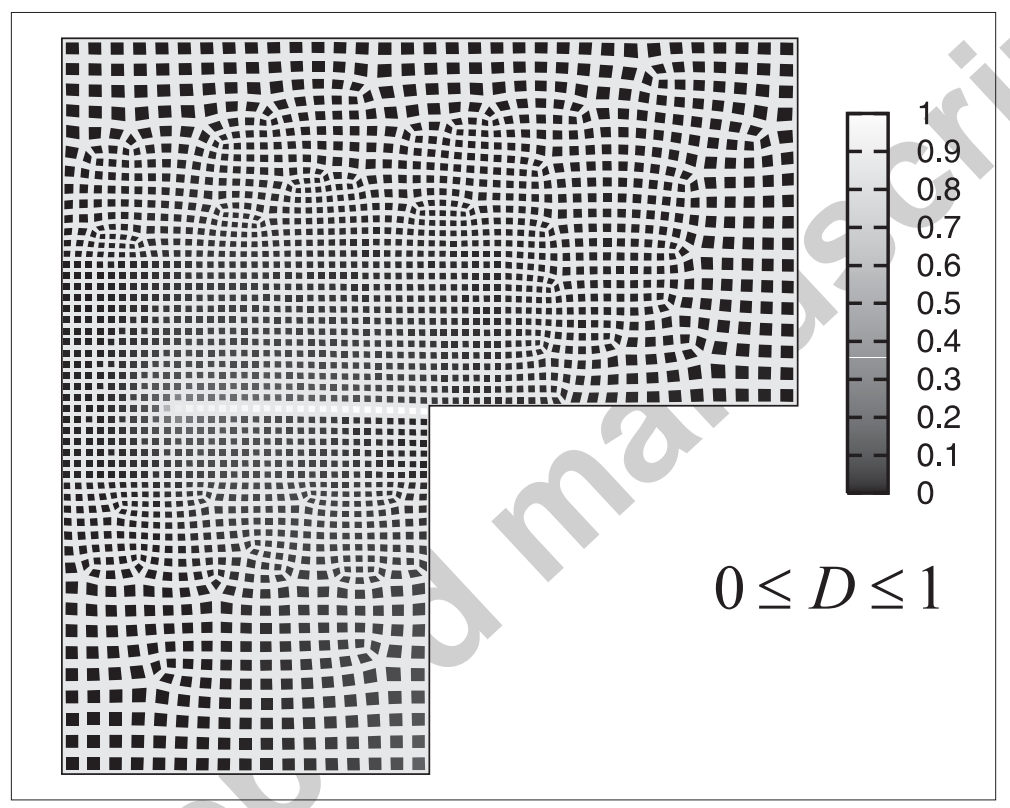

P.

(1)

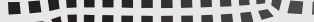

10

$0 \leq D \leq 1$

(1)

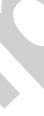




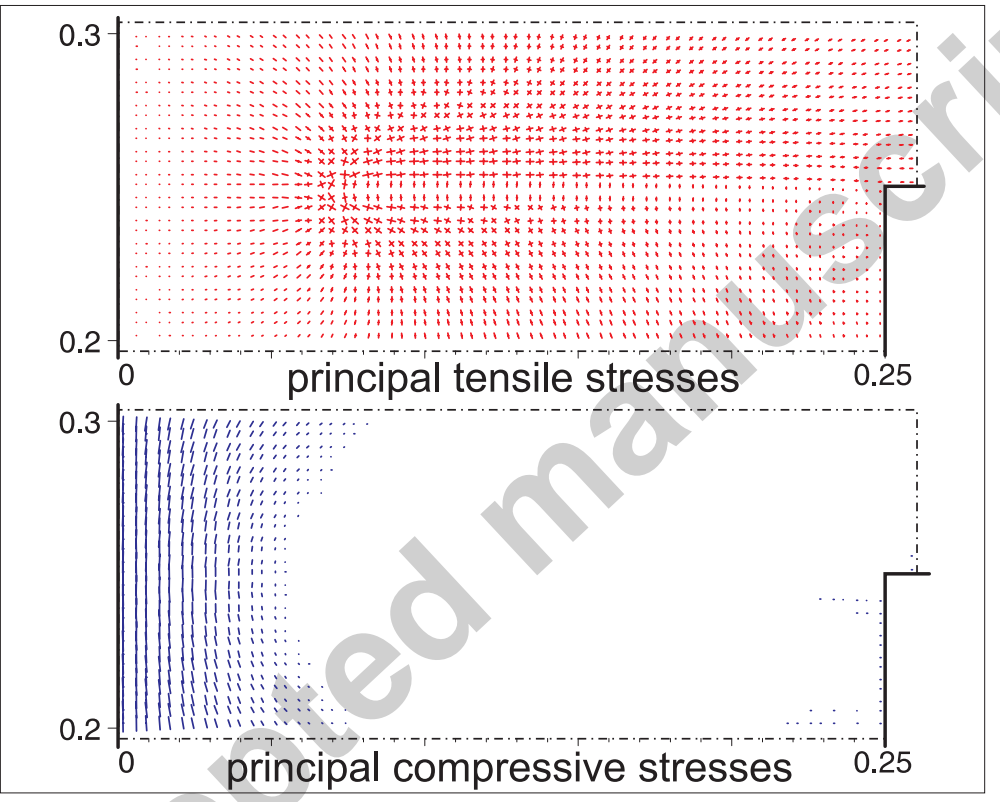




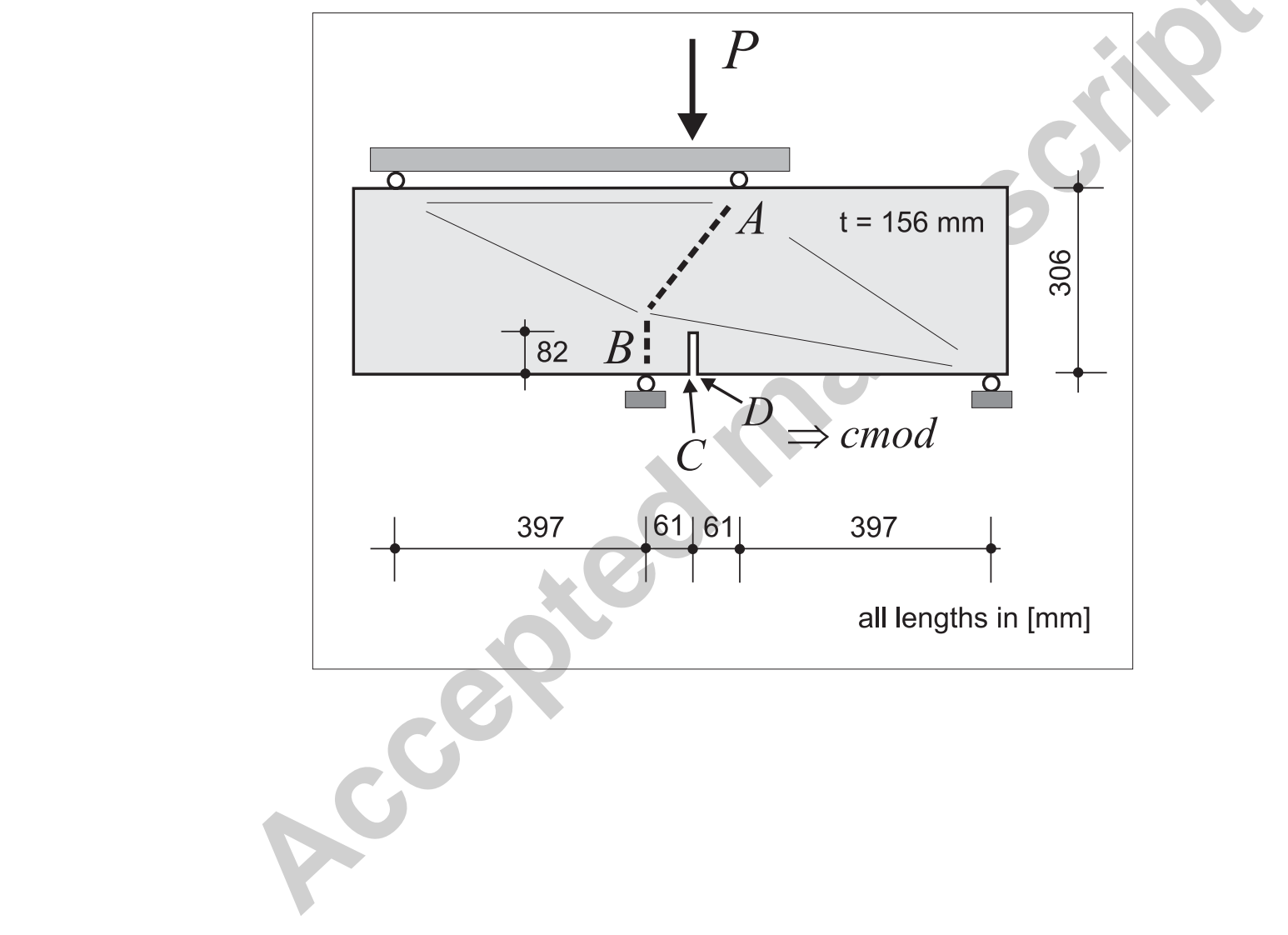

Figure 14

(1) all lengths in [mm

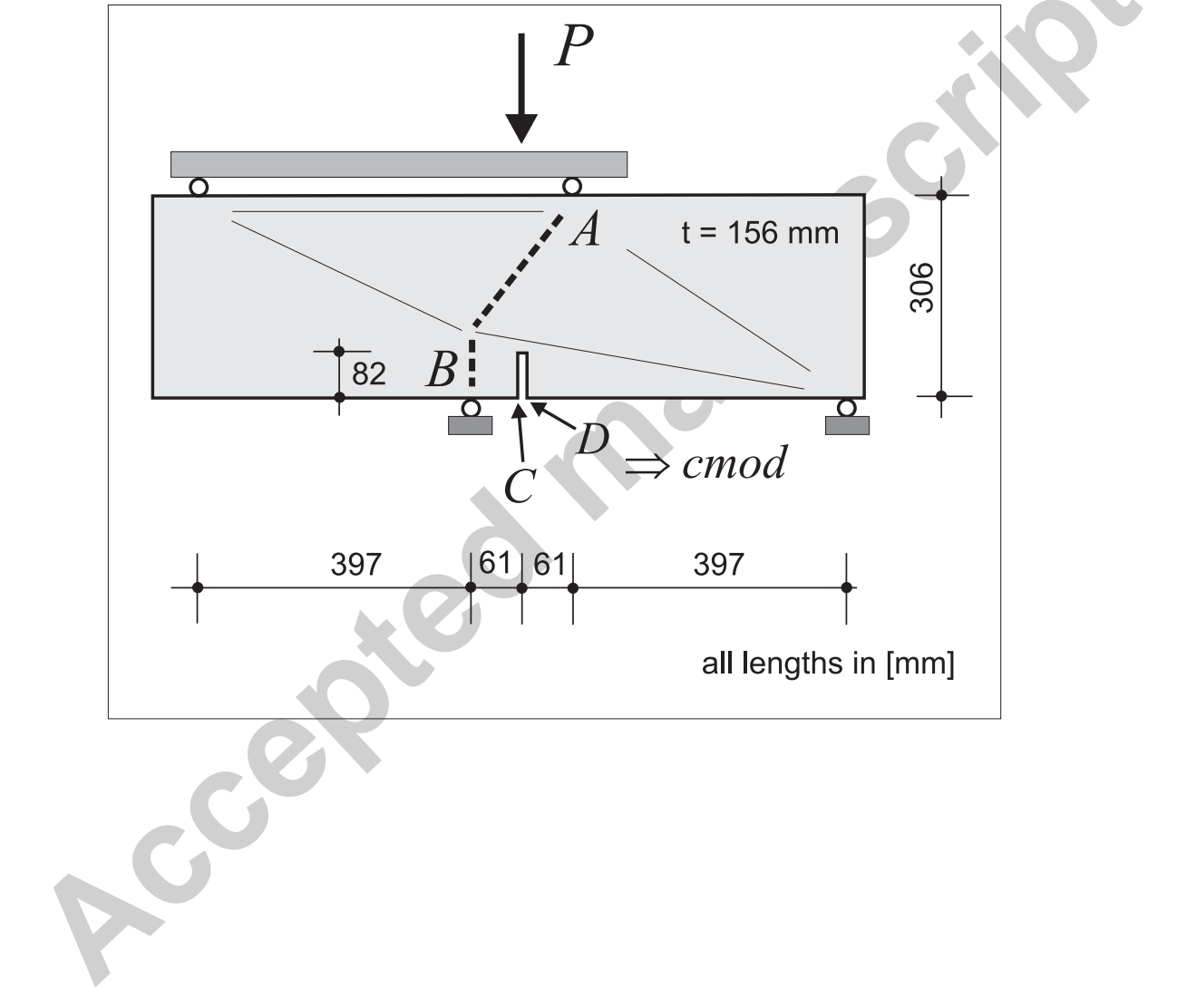

-

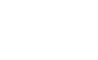

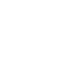

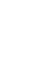




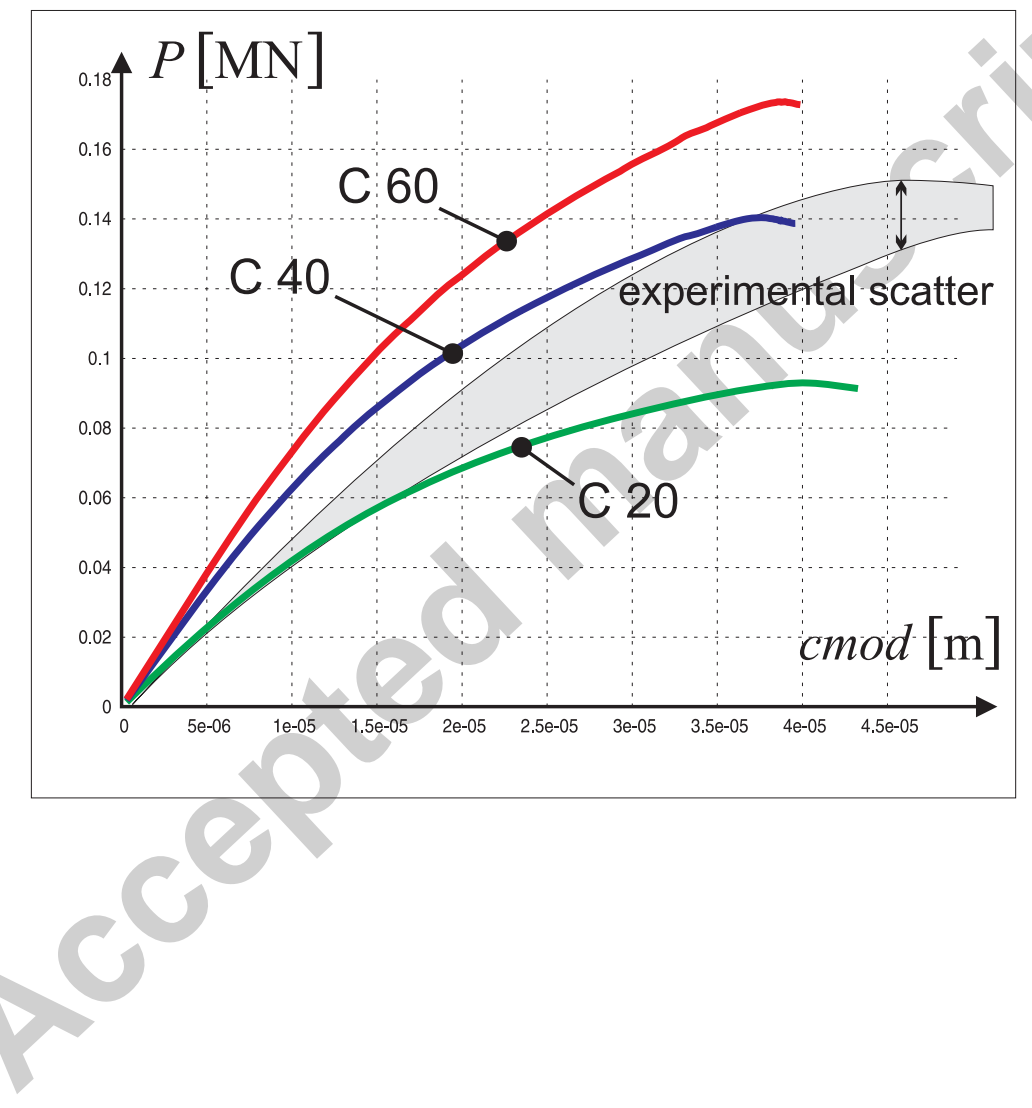

Figure 15

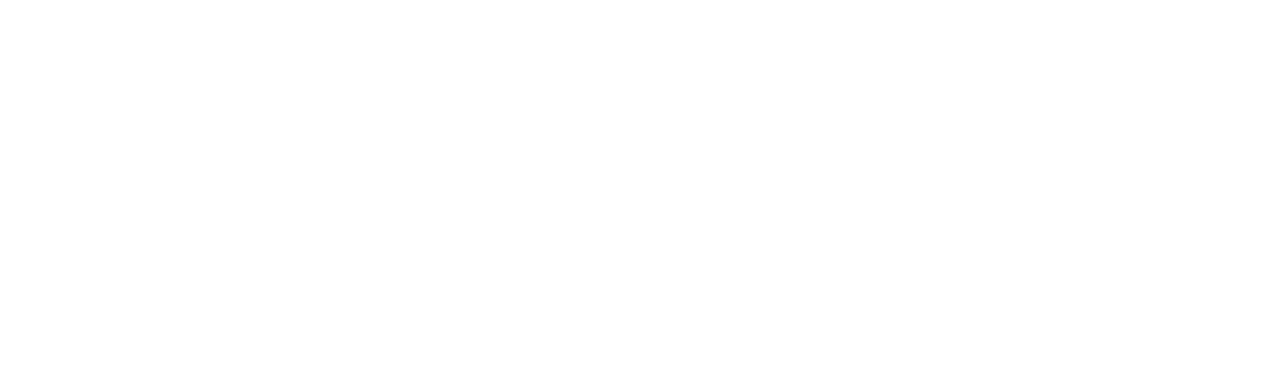

Fis 


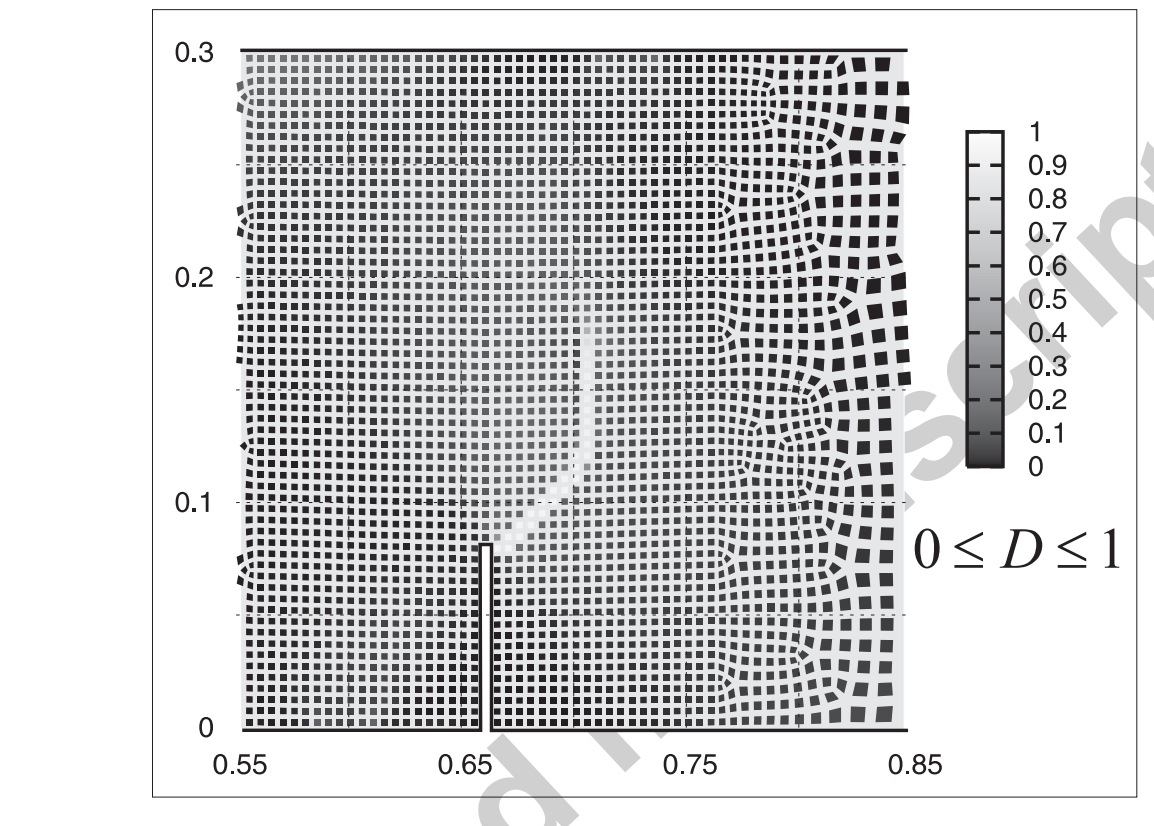




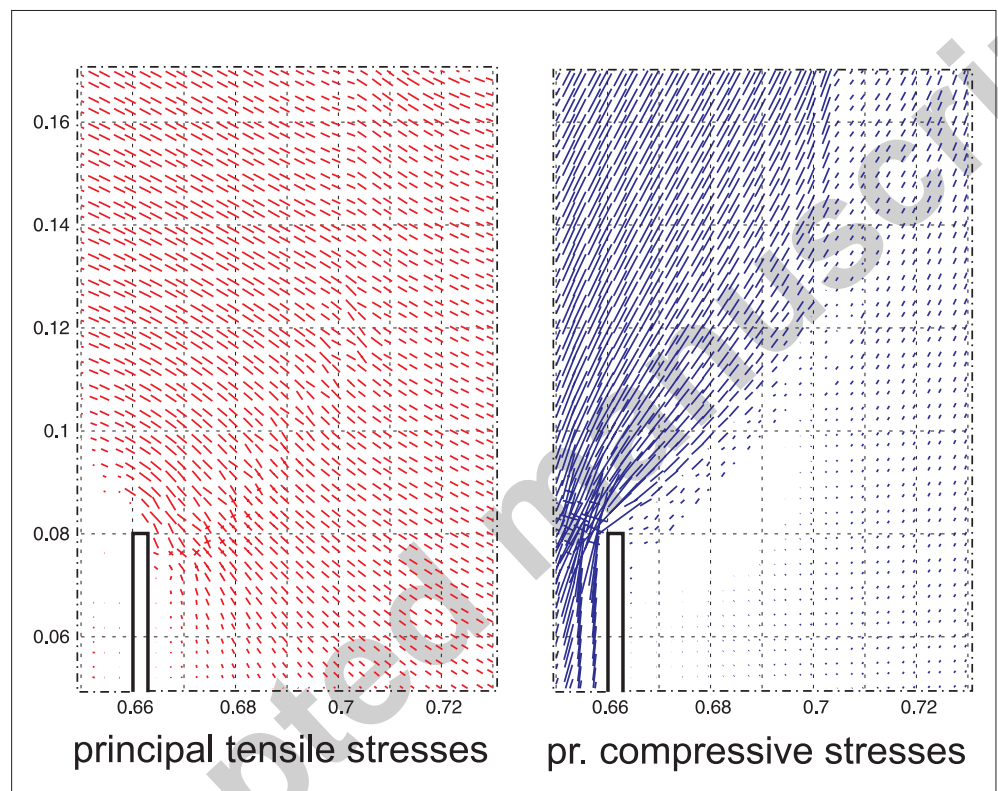

Figure 17

\begin{abstract}
pr. compressive stresses
\end{abstract}

